The Astrophysical Journal, 673:128-142, 2008 January 20

(C) 2008. The American Astronomical Society. All rights reserved. Printed in U.S.A.

\title{
RADIO CONTINUUM JET IN NGC 7479
}

\author{
SePpo Laine \\ Spitzer Science Center, California Institute of Technology, MS 220-6, Pasadena, CA 91125; seppo@ipac.caltech.edu \\ AND \\ RAINER BECK \\ Max-Planck-Institut für Radioastronomie, 53121 Bonn, Germany; rbeck@mpifr-bonn.mpg.de \\ Received 2007 June 20; accepted 2007 September 26
}

\begin{abstract}
The barred galaxy NGC 7479 hosts a remarkable jetlike radio continuum feature: bright, $12 \mathrm{kpc}$ long in projection, and hosting an aligned magnetic field. The degree of polarization is $6 \%-8 \%$ along the jet and remarkably constant, which is consistent with helical field models. The radio brightness of the jet suggests strong interaction with the ISM and hence a location near the disk plane. We observed NGC 7479 at four wavelengths with the VLA and Effelsberg radio telescopes. The equipartition strength is $35-40 \mu \mathrm{G}$ for the total and $\geq 10 \mu \mathrm{G}$ for the ordered magnetic field in the jet. The jet acts as a bright, polarized background. Faraday rotation between 3.5 and $6 \mathrm{~cm}$ and depolarization between 6 and $22 \mathrm{~cm}$ can be explained by magnetoionic gas in front of the jet, with thermal electron densities of $\simeq 0.06 \mathrm{~cm}^{-3}$ in the bar and $\simeq 0.03 \mathrm{~cm}^{-3}$ outside the bar. The regular magnetic field along the bar points toward the nucleus on both sides. The regular field in the disk reveals multiple reversals, probably consisting of field loops stretched by a shearing gas flow in the bar. The projection of the jet bending in the sky plane is in the sense opposite to that of the underlying stellar and gaseous spiral structure. The bending in $3 \mathrm{D}$ is most easily explained as a precessing jet, with an age less than $10^{6} \mathrm{yr}$. Our observations are consistent with very recent triggering, possibly by a minor merger. NGC 7479 provides a unique opportunity to study interaction-triggered $15 \mathrm{kpc}$ scale radio jets within a spiral galaxy.
\end{abstract}

Subject headings: galaxies: active — galaxies: nuclei — galaxies: Seyfert — galaxies: starburst — radio continuum: galaxies

\section{INTRODUCTION}

Radio jets are commonly seen around radio galaxies, which themselves are usually associated with distant elliptical galaxies (Bridle \& Perley 1984). Relatively few examples of radio galaxies are known at distances less than $100 \mathrm{Mpc}$ from us. The radio jets in these systems can have lengths up to a few megaparsecs. The radio continuum synchrotron emission comes from relativistic electrons spiraling in magnetic fields. The origin of the power in radio galaxies is tied to energetic events taking place near their nuclei, most likely ejections or outflows from an accretion disk surrounding a massive nuclear black hole (e.g., Rees et al. 1982).

Smaller scale jets are often seen near the nuclei of Seyfert galaxies (e.g., Ho \& Ulvestad 2001 and references therein). In these objects the jets have lengths from less than a parsec to a few kiloparsecs. Again, the energy source is probably near the nuclear black hole, this time 10-1000 times less massive than the black holes in radio galaxies. For example, NGC 3079 hosts a nuclear radio jet feature within the central parsec (Irwin \& Seaquist 1988) and bipolar radio lobes extending out to $3 \mathrm{kpc}$, perpendicular to the edge-on galaxy plane (Duric \& Seaquist 1988). The Circinus spiral galaxy shows similar kiloparsec-scale (projected) bipolar radio lobes (Harnett et al. 1990) and a projected $100 \mathrm{pc}$ scale jetlike feature aligned with the radio lobes (Elmouttie et al. 1998), together with a parsec-scale nuclear outflow (Greenhill et al. 2003). The spiral galaxy 0313-192 hosts a one-sided nuclear jet of about $2 \mathrm{kpc}$ length that powers bright radio lobes of type FR I extending to about $100 \mathrm{kpc}$ from the nucleus (Ledlow et al. 2001; Keel et al. 2006).

Curiously, there exist relatively few nearby galaxies with large jets on the $10 \mathrm{kpc}$ scale. Disk galaxies with such jets are even less common. In fact, the only examples of $15 \mathrm{kpc}$ scale anomalous radio arms or double-sided radio jets in disk galaxies in the nearby universe are in NGC 4258 (e.g., Hummel et al. 1989; Krause \& Löhr 2004) and NGC 7479 (Laine \& Gottesman 1998). The radio jet in NGC 4258 is detected out to about $14 \mathrm{kpc}$ radius and is of spiral form, trailing with respect to the galaxy rotation. It is clearly interacting with the dense interstellar medium (ISM) gas in the galactic plane, evidenced by similar structures seen in $\mathrm{H} \alpha$ and X-ray emission (e.g., Wilson et al. 2001) and a peculiar distribution of the CO line emission in the disk (Krause et al. 1990, 2007). Numerous investigations have been carried out on the nature of the jet in NGC 4258 (e.g., Cecil et al. 1995, 2000; Cox \& Downes 1996; Herrnstein et al. 1997; Vogler \& Pietsch 1999; Wilson et al. 2001). Wilson et al. (2001) argue that the interaction could be indirect via halo gas heated by the jet and falling back onto the disk, so that the jet itself may be extraplanar. In contrast to the jet in NGC 4258 , the bright, jetlike radio continuum structure in NGC 7479 has remained practically unnoticed since its serendipitous discovery as a by-product of the $\mathrm{H}$ I observations of Laine \& Gottesman (1998).

NGC 7479 is a relatively nearby (distance $\approx 32 \mathrm{Mpc}$ ) SBc type spiral galaxy. Table 1 gives some basic properties of NGC 7479. The nucleus has been classified as a LINER (Keel 1983) and as a Seyfert 1.9 (Ho et al. 1997). The Chandra X-ray observations by Panessa et al. (2006) revealed an X-ray luminosity of $1.3 \times 10^{41} \mathrm{ergs} \mathrm{cm}^{-2} \mathrm{~s}^{-1}$ in the $2-10 \mathrm{keV}$ band. The inferred nuclear black hole mass is $1.2 \times 10^{7} \mathrm{M}_{\odot}$, which is almost as large as the nuclear black hole in NGC $4258\left(4 \times 10^{7} M_{\odot}\right.$; Miyoshi et al. 1995; Greenhill et al. 1995a, 1995b; Herrnstein et al. 1999). The X-ray luminosity of NGC 4258 in the $2-10 \mathrm{keV}$ band is $7.2 \times 10^{40} \mathrm{ergs} \mathrm{cm}^{-2} \mathrm{~s}^{-1}$ (Panessa et al. 2006), very close to the 
TABLE 1

Basic Parameters of NGC 7479

\begin{tabular}{|c|c|}
\hline Parameter & Value \\
\hline Galaxy type....... & $\operatorname{SBbc}(\mathrm{s})$ \\
\hline Environment.... & Isolated \\
\hline Right ascension (J2000.0) ... & 230456.65 \\
\hline 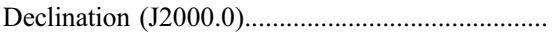 & 121922.4 \\
\hline 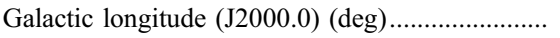 & 86.2711 \\
\hline 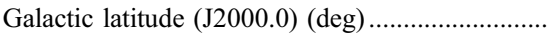 & -42.8417 \\
\hline 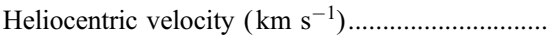 & 2371 \\
\hline Distance $(\mathrm{Mpc})$ & 32 \\
\hline Linear scale & $1^{\prime \prime} \cong 160 \mathrm{pc}$ \\
\hline 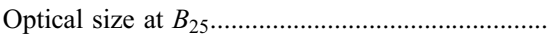 & $4.1^{\prime} \times 3.1^{\prime}$ \\
\hline Inclination $(\mathrm{deg}) . . .$. & 51 \\
\hline Position angle of the disk major axis $(\mathrm{deg}) \ldots \ldots \ldots . .$. & 22 \\
\hline 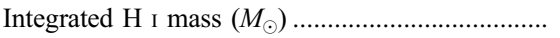 & $8.6 \times 10^{9}$ \\
\hline $\mathrm{H}_{2}$ mass $\left(M_{\odot}\right)$ & $2.5 \times 10^{10}$ \\
\hline 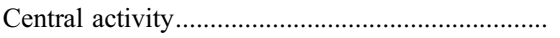 & LINER; Sy 1.9; starburst \\
\hline 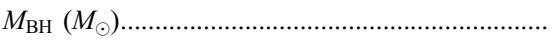 & $1.2 \times 10^{7}$ \\
\hline 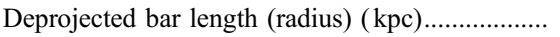 & 8.6 \\
\hline Position angle of bar (deg).. & 6 \\
\hline
\end{tabular}

Notes.- - Units of right ascension are hours, minutes, and seconds, and units of declination are degrees, arcminutes, and arcseconds. Galaxy type from Sandage \& Tammann (1987), right ascension and declination from NED, heliocentric velocity from Laine \& Gottesman (1998), distance assuming Hubble law with $H_{0}=$ $75 \mathrm{~km} \mathrm{~s}^{-1}$, size from NED, inclination, position angle, and $\mathrm{H}$ I mass from Laine \& Gottesman (1998), $\mathrm{H}_{2}$ mass from Young et al. (1989), LINER classification from Keel (1983), Seyfert 1.9 classification from Ho et al. (1997), starburst classification from Devereux (1989), black hole mass from Panessa et al. (2006), bar length from Blackman (1983), position angle of the bar from Burbidge et al. (1960).

value measured for NGC 7479. Therefore, the $L_{\mathrm{X}} / M_{\mathrm{BH}}$ ratio is about a factor of 6 higher in NGC 7479 compared to NGC 4258. The VLA observations of NGC 7479 at $2 \mathrm{~cm}$ with $\simeq 0.15^{\prime \prime}$ resolution (Nagar et al. 2005) show a $2.5 \mathrm{mJy}$ beam $^{-1}$ nuclear source in NGC 7479. VLA observations at 6 and $20 \mathrm{~cm}$ with $\simeq 1^{\prime \prime}$ resolution reveal an extended feature pointing from the nucleus toward the northwest (Ho \& Ulvestad 2001; Laine et al. 2006), coinciding with the inner part of the radio jet (see below). Optical Hubble Space Telescope (HST) images (G. F. Benedict 1996, private communication) show a complicated dust structure with a stronger dust lane leading into the nucleus from north (apparently an inner extension of the leading bar dust lane).

At larger scales, there are many signs of a recent perturbation in the disk of NGC 7479. These include the strongly asymmetric spiral structure, consisting of a strong and long western spiral arm that appears to bifurcate west of the nucleus, while there is little organized spiral structure on the eastern side (e.g., Quillen et al. 1995). Further signatures of a recent perturbation include the irregular $\mathrm{H}$ i velocity field along the western arm (Laine \& Gottesman 1998), a peculiar perturbation in the $\mathrm{H} \alpha$ velocity field north of the nucleus (Laine \& Heller 1999), anomalous dust lane structure around the bar as seen in the optical images (Laine 1996), and emission-line excitation asymmetries (Martin et al. 2000). These characteristics have led Quillen et al. (1995) and Laine \& Heller (1999) to suggest that the galaxy has recently suffered a minor merger with a sizable companion. No companions have been found with optical imaging (Saraiva \& Benedict 2003) or H I observations within a radius of several tens of kiloparsecs around NGC 7479 (Laine \& Gottesman 1998). There is no visible trace of a remnant of a merging companion either, but the minor merger models of Laine \& Heller (1999) predict that the nuclear remnant of a mostly disrupted companion should currently be in the bar, north of the nucleus, coinciding with the per- turbation in the $\mathrm{H} \alpha$ velocity field. The strong stellar bar was also proposed to have formed as a result of the minor merger.

The first radio continuum polarization observations of $\mathrm{NGC}$ 7479 by Beck et al. (2002) with the VLA at several wavelengths with $30^{\prime \prime}$ resolution could not resolve the jet but showed a curious winglike pattern at $6 \mathrm{~cm}$, extending perpendicular to the bar, with magnetic field lines oriented parallel to the bar.

This paper reports observations of polarized radio continuum emission from NGC 7479, and specifically on the anomalous jetlike radio continuum structure that has a total projected extent of around $12 \mathrm{kpc}$ and forms a spiral that leads with respect to the galaxy rotation. In other words, the radio continuum feature bends in the opposite sense to the stellar and gaseous spiral arms. Since its discovery by Laine \& Gottesman (1998), no evidence of this feature at any other spectral regime has been found. This suggests that the feature is not interacting with the gas in the disk of NGC 7479 and likely extends (at least partly) out of the plane, while its high radio brightness indicates strong interaction with the ISM in the disk. We present total intensity and polarized emission data that further suggest a jet origin for the anomalous radio continuum feature. The origin, orientation, and nature of the jet are examined and compared to radio jets seen in other nearby galaxies. We also investigate the nuclear point source, the bar region, the extended radio continuum emission from NGC 7479, and the nature of point sources within and outside the optical disk with the help of polarization measurements.

\section{OBSERVATIONS}

VLA observations of NGC 7479 were taken during several separate observing sessions ( Table 2). Weather conditions were generally good during the observations. Absolute flux calibration is based on scans of 3C 286 and 3C 138, which also served as absolute polarization angle calibrators. The target galaxy observations were bracketed between 1-2 minute scans of the nearby phase calibrator $\mathrm{J} 2253+1608$, and their fluxes were bootstrapped to those of the primary calibrators. Uncertainties in the fluxes quoted in the VLA Calibrator Manual are dominated by the uncertainty in setting the absolute flux scale and are estimated to be $\approx 5 \%$. Uncertainties in the positions are estimated to be less than $0.1^{\prime \prime}$. Uncertainties in the absolute value of the polarization angle are estimated to be less than $1^{\circ}$.

For maximum sensitivity we used two intermediate frequencies (IFs), with bandwidths of $50 \mathrm{MHz}$ and separated by $50 \mathrm{MHz}$, except in the case of 18 and $22 \mathrm{~cm}$ observations when we used only one IF at each wavelength. After minimal editing of the visibilities and calibration, we used the AIPS task IMAGR to Fourier transform the observed visibilities into brightness distribution maps on the sky. We applied phase-only self-calibration to the $6 \mathrm{~cm} \mathrm{C}$ configuration data, resulting in a slight decrease in rms noise. Final cleaning was commonly performed for 10,000 iterations, except in the highest resolution maps, in which we cleaned for 50,000 iterations. Varying the value of the ROBUST parameter, maps with different resolutions were made. The best resolution of the $\mathrm{C}$ configuration data is $2.2^{\prime \prime}$ (synthesized halfpower beamwidth) at $3.5 \mathrm{~cm}$ and about $4^{\prime \prime}$ at $6 \mathrm{~cm}$. These maps were subsequently smoothed to circular beams of $6^{\prime \prime}, 8^{\prime \prime}$, and $12^{\prime \prime}$. The $\mathrm{D}$ configuration data at $3.5 \mathrm{~cm}$ give a best resolution of $8^{\prime \prime}$, with an rms noise about half that of the smoothed map obtained from the $\mathrm{C}$ configuration data. We did not find substantial improvement in maps made from combined C and D configuration $u v$ data and chose to use the $3.5 \mathrm{~cm}$ images made with data from separate configurations. No correction for the primary beam attenuation was made. The $\mathrm{D}$ configuration data 
TABLE 2

Parameters of the Observing Runs

\begin{tabular}{|c|c|c|c|c|}
\hline Date & Telescope & $\begin{array}{l}\text { Observation Time } \\
\text { (s) }\end{array}$ & $\begin{array}{l}\text { Wavelength } \\
\text { (cm) }\end{array}$ & Notes \\
\hline $1996 \operatorname{Mar} 22 .$. & VLA-C & 2400 & 18,22 & Beck et al. (2002) \\
\hline 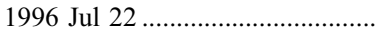 & VLA-D & 2200 & 3.5 & Beck et al. (2002) \\
\hline 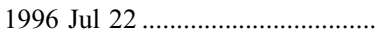 & VLA-D & 1800 & 6.2 & Beck et al. (2002) \\
\hline 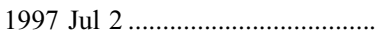 & VLA-C & 5300 & 18,22 & Beck et al. (2002) \\
\hline 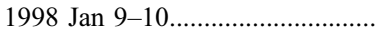 & VLA-D & 19500 & 3.5 & \\
\hline 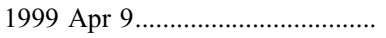 & VLA-D & 8500 & 6.2 & Hardware failure \\
\hline 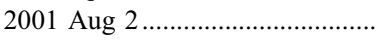 & VLA-C & 25500 & 3.5 & \\
\hline 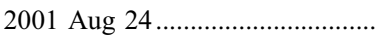 & VLA-C & 24790 & 6.2 & \\
\hline 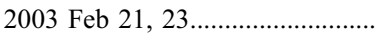 & Effelsberg & 12000 & 3.6 & \\
\hline
\end{tabular}

at $6 \mathrm{~cm}$ from Beck et al. (2002), obtained from 30 minutes of total observing time, are not sensitive enough for the purposes of this paper. The more recent $\mathrm{D}$ configuration data at $6 \mathrm{~cm}$ could not be used due to a severe hardware problem during the observations, so that only the $C$ configuration data at $6 \mathrm{~cm}$ were used for this paper.

We made maps in the Stokes parameters $I, Q$, and $U$. The largest structures visible in our observations are about $3^{\prime}$ at $3.5 \mathrm{~cm}$ and $5^{\prime}$ at $6 \mathrm{~cm}$. Hence, our maps can be used reliably to study the spectral index only in the central bright parts, including the jetlike structure. It is unlikely that any large-scale structure is missing in the Stokes parameters $Q$ and $U$, since the polarization angle changes throughout the galaxy. We made maps of the linearly polarized intensity (PI) and the polarization angle (P.A.) from the $Q$ and $U$ maps, adding $90^{\circ}$ to obtain $B$-vectors. The positive bias in PI was corrected with the POLCO routine in AIPS.

To map the largest structures, $3.6 \mathrm{~cm}$ observations of NGC 7479 were performed in 2003 February under excellent weather conditions with the Effelsberg single-dish telescope using the $8.35 \mathrm{GHz}$ receiver with $1.1 \mathrm{GHz}$ bandwidth and a beam size of $84^{\prime \prime}$. A field of $10^{\prime} \times 10^{\prime}$ was scanned alternately in right ascension and declination. Eleven coverages were combined using the standard NOD2 software package. The final maps in Stokes $I$, $Q$, and $U$ were smoothed to $90^{\prime \prime}$ resolution. The rms noise is $\simeq 400 \mu \mathrm{Jy}_{\text {beam }}{ }^{-1}$ in total intensity (limited by scanning effects) and $70 \mu \mathrm{Jy}$ beam $^{-1}$ in $Q$ and $U$. A combination of VLA and Effelsberg $3 \mathrm{~cm}$ data was not attempted as we concentrate on the jet properties in this paper.

\section{RESULTS}

\subsection{Total Intensity and Spectral Index}

A VLA contour map of the total radio intensity at $2.2^{\prime \prime}$ resolution at $3.5 \mathrm{~cm}$ is shown in Figure 1, and maps of the total radio intensity at $8^{\prime \prime}$ resolution at 3.5 and $6 \mathrm{~cm}$ are shown in Figure 2. The S-shaped, double-jet feature is the dominant structure. There is little indication (lack of detailed spatial correlation with bar structure in high-resolution images) that a considerable fraction of the radio continuum emission emerges from the bar region (at $+6^{\circ}$ position angle), although we cannot exclude this possibility. The emission along the jet is asymmetric, with the northern side being stronger in total intensity by about $30 \%$ in the $8^{\prime \prime}$ maps at 3.5 and $6 \mathrm{~cm}$. At the highest resolution (Fig. 1) the shape of the northern jet is also more regular and narrower than the southern jet, which breaks into diffuse clouds separated by apparent gaps due to the low signal-to-noise ratio at this resolution. The full width at half-power of the northern jet is $2.7^{\prime \prime}$. Its physical width, corrected for beam smearing, is about $250 \mathrm{pc}$. At $8^{\prime \prime}$ resolution (Fig. 2) the southern jet is also continuous, but with an intensity minimum at about $17^{\prime \prime}$ from the center. The projected jet lengths in Figure 2 are 5.5 and $6.5 \mathrm{kpc}$ in the north and south, respectively.

Radio emission from the bar and the adjacent spiral arms is also detected (Fig. 2), but it is much weaker than the emission from the jet. Possibly a fraction of the radio continuum emission along the inner jet comes from the bar component, related to amplified magnetic fields in the shocks of the bar (Beck et al. 2005). We tried to subtract this component using Spitzer observations and a known far-IR-radio continuum correlation, but unfortunately the resolution of the far-IR Spitzer observations is too low (close to $20^{\prime \prime}$ at $70 \mu \mathrm{m}$ ) and there is a strong nuclear point source with extended diffraction structure that is difficult to remove and that makes the estimation of a bar component even harder.

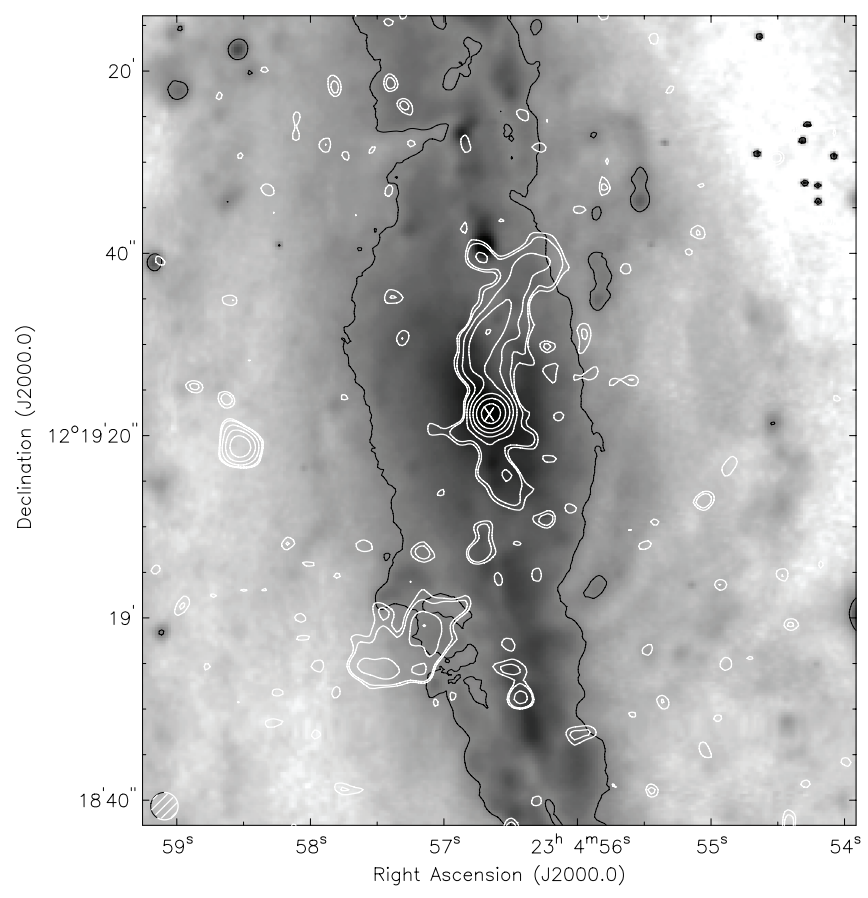

FIG. 1.-Contour (white) image of the $3.5 \mathrm{~cm}$ total intensity (Stokes $I$ ) emission from the center of NGC 7479 at $2.2^{\prime \prime}$ resolution (C configuration), overlaid on an optical $B$-band image. The nucleus is marked with a white cross, and the $22 \mathrm{mag} \operatorname{arcsec}^{-2}$ isophote on the $B$ image is drawn with a black contour to show the stellar bar. The rms noise is $13 \mu \mathrm{Jy}$ beam $^{-1}$. The contour levels are at $(3,4,8,16,32,64,128) \times 11 \mu \mathrm{Jy}_{\text {beam }}{ }^{-1}$. No polarized emission is detected at this resolution. The beam is shown at the bottom left corner. 


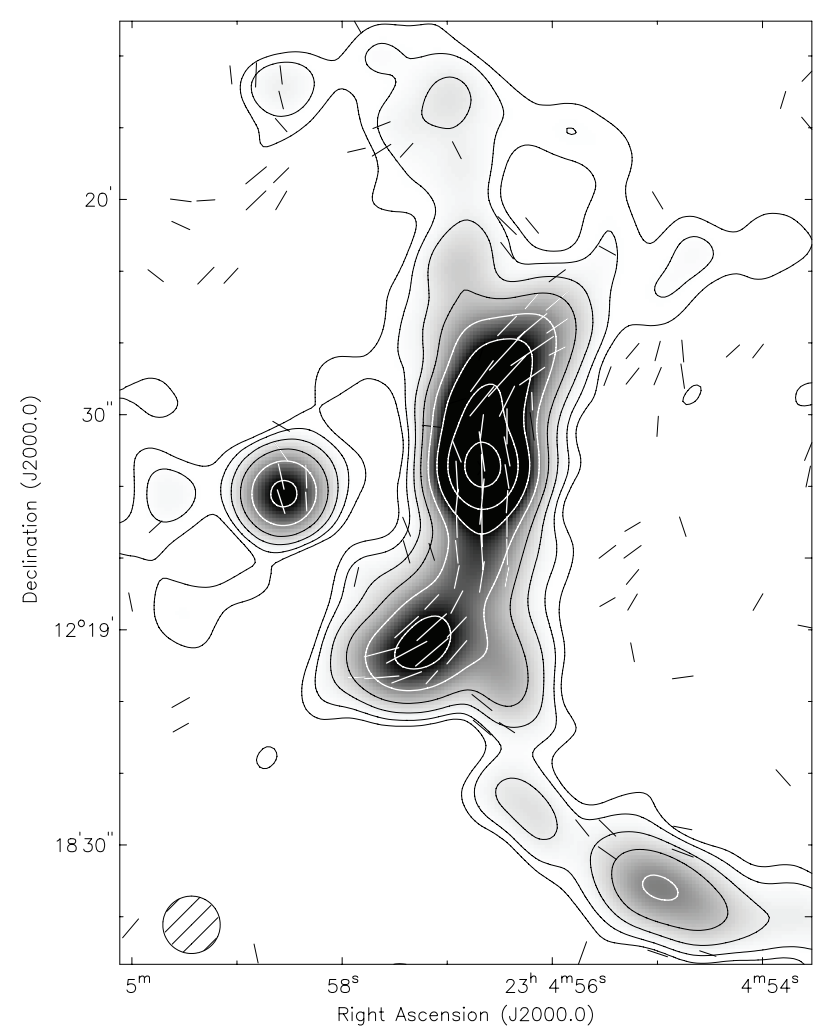

FIG. $2 a$

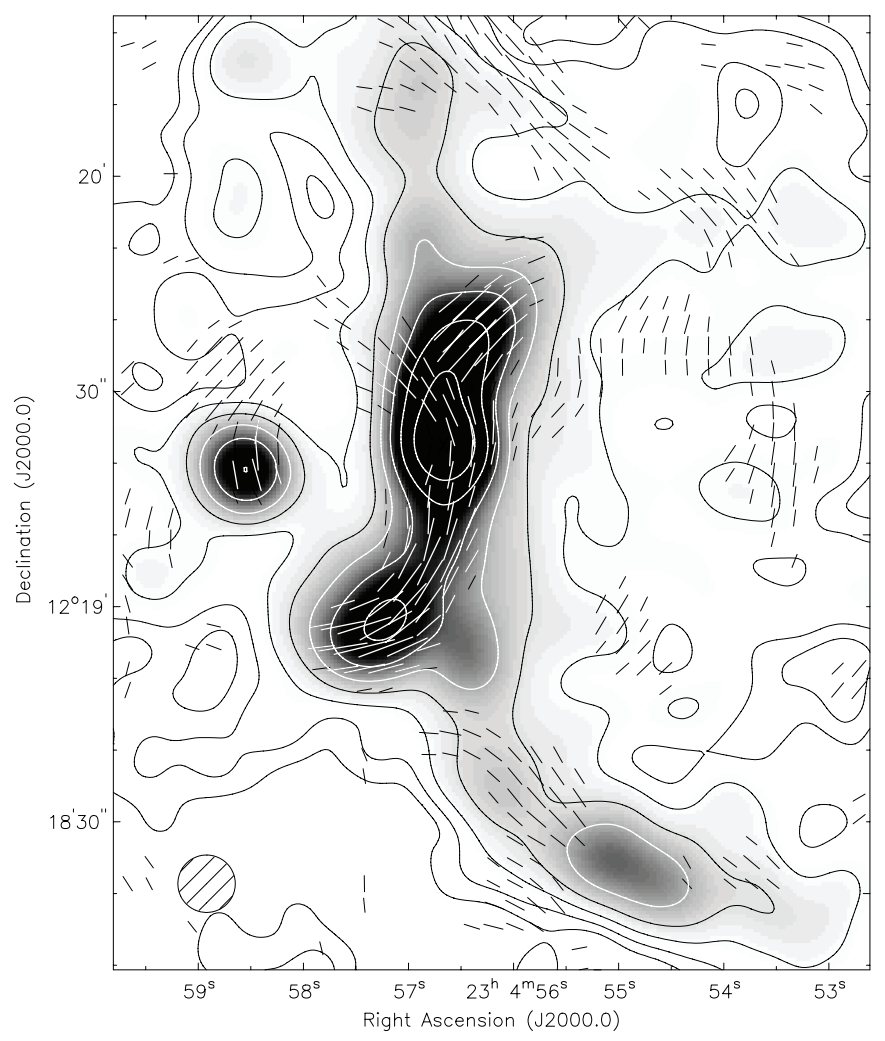

FIG. $2 b$

FIg. 2.- (a) $B$-vectors $\left(E\right.$-vectors rotated by $90^{\circ}$ ) overlaid on a gray-scale and contour image of the $3.5 \mathrm{~cm}$ total intensity at $8^{\prime \prime}$ resolution (D configuration). The rms noise is $11 \mu \mathrm{Jy}_{\text {beam }}{ }^{-1}$. The contours are at $(2,4,8,16,32,64,128,256) \times 12 \mu \mathrm{Jy}_{\text {beam }}^{-1}$. The telescope beam is shown at the bottom left corner. The nucleus is shown with a white cross. (b) $B$-vectors ( $E$-vectors rotated by $90^{\circ}$, not corrected for Faraday rotation) overlaid on a gray-scale and contour image of the $6 \mathrm{~cm}$ total intensity at $8^{\prime \prime}$ resolution (C configuration). The rms noise is $9 \mu \mathrm{Jy}_{\text {beam }}{ }^{-1}$. The contours are at $(2,4,8,16,32,64,128,256) \times 10 \mu \mathrm{Jy}$ beam ${ }^{-1}$. The telescope beam is shown close to the bottom left corner. The nucleus is shown with a white cross.

The spectral index map at 8 " resolution in Figure 3 shows that the spectral index between 3.5 and $6 \mathrm{~cm}$ is close to $-0.5 \pm 0.03$ near the nucleus and $-0.8 \pm 0.04$ along the jet. (Although the cited uncertainties are valid for the absolute values of the spectral indices, the uncertainty in relative values between different locations in the image is smaller, since all the pixels in a given image are subject to common calibration uncertainties. The uncertainty in the relative spectral index between adjacent regions in the image is determined by the rms noise values in the individual images and is approximately \pm 0.01 near the nucleus and approximately \pm 0.03 in the jet.) As thermal emission from the nucleus and the jet is probably small, the above values can be taken as the synchrotron spectral index. No significant steepening is observed along the jet, which indicates fast propagation of cosmic rays along the jet. The mean value of the spectral index between 3.5 and $6 \mathrm{~cm}$ in the southern spiral arm is around -0.75 . Assuming a typical thermal fraction of $25 \%$ at $6 \mathrm{~cm}$, the synchrotron spectral index is $\simeq-1.0$.

The image at $12^{\prime \prime}$ resolution (Fig. 4) has high sensitivity to diffuse extended emission. The two optical/near-infrared spiral arms emerging from the ends of the bar, specifically the southwestern arm, are also seen in the total radio intensity map. Clouds of diffuse radio emission west of the bar (between the bar and the outer spiral arm) and also east of the bar indicate a reservoir of relativistic electrons and magnetic fields. In the Effelsberg $3.6 \mathrm{~cm}$ map (Fig. 5) and in the VLA maps at 18 and $20 \mathrm{~cm}$ wavelengths (Beck et al. 2002) diffuse emission in total intensity maps can be followed out to about $23 \mathrm{kpc}$ projected radius.

\subsection{Polarized Intensity}

We present $8^{\prime \prime}$ resolution maps of the polarized emission at 3.5 and $6 \mathrm{~cm}$ in Figure 6. The strongest polarized emission emerges from blobs at the jet ends. Polarized intensity appears to be somewhat higher at $6 \mathrm{~cm}$ than at $3.5 \mathrm{~cm}$. However, as the signal-to-noise ratio at $3.5 \mathrm{~cm}$ is worse than at $6 \mathrm{~cm}$, the intensity differences are hardly significant (while the angle difference is significant; see below).

The position angles of the $B$-vectors at $3.5 \mathrm{~cm}$ remain almost constant $\left(140^{\circ}-180^{\circ}\right)$ along the jet but decrease to $\approx 90^{\circ}$ toward the southern end of the jet and to $\approx 45^{\circ}$ toward the northern end, giving an indication of bending of the jet at its ends.

The polarized emission at $6 \mathrm{~cm}$ in the outer northern jet emerges mostly from a source near the end of the jet at R.A.J2000.0 $=$ $23^{\mathrm{h}} 04^{\mathrm{m}} 56.3^{\mathrm{s}}$, decl. J2000.0 $=12^{\circ} 19^{\prime} 39^{\prime \prime}$, which is barely visible in the maps of total emission. No source is seen at this location in the optical and infrared maps, only a dust lane extending toward south-southwest. The polarized emission at $3.5 \mathrm{~cm}$ is elongated along the outer northern jet, with only a small gap toward the inner jet. The $B$-vectors at $6 \mathrm{~cm}$ (Fig. $6 b$ ) in the northern jet, south of the polarized source, turn toward the northeast due to strong Faraday rotation between 3.5 and $6 \mathrm{~cm}$, while Faraday rotation in the polarized source is smaller (see $\S 3.4$ ). Hence, the gap at $6 \mathrm{~cm}$ between the polarized source and the inner jet is due to Faraday depolarization.

The degree of polarization is remarkably constant along the jet, with values between $6 \%$ and $8 \%$ at $3.5 \mathrm{~cm}$. Lower values near 


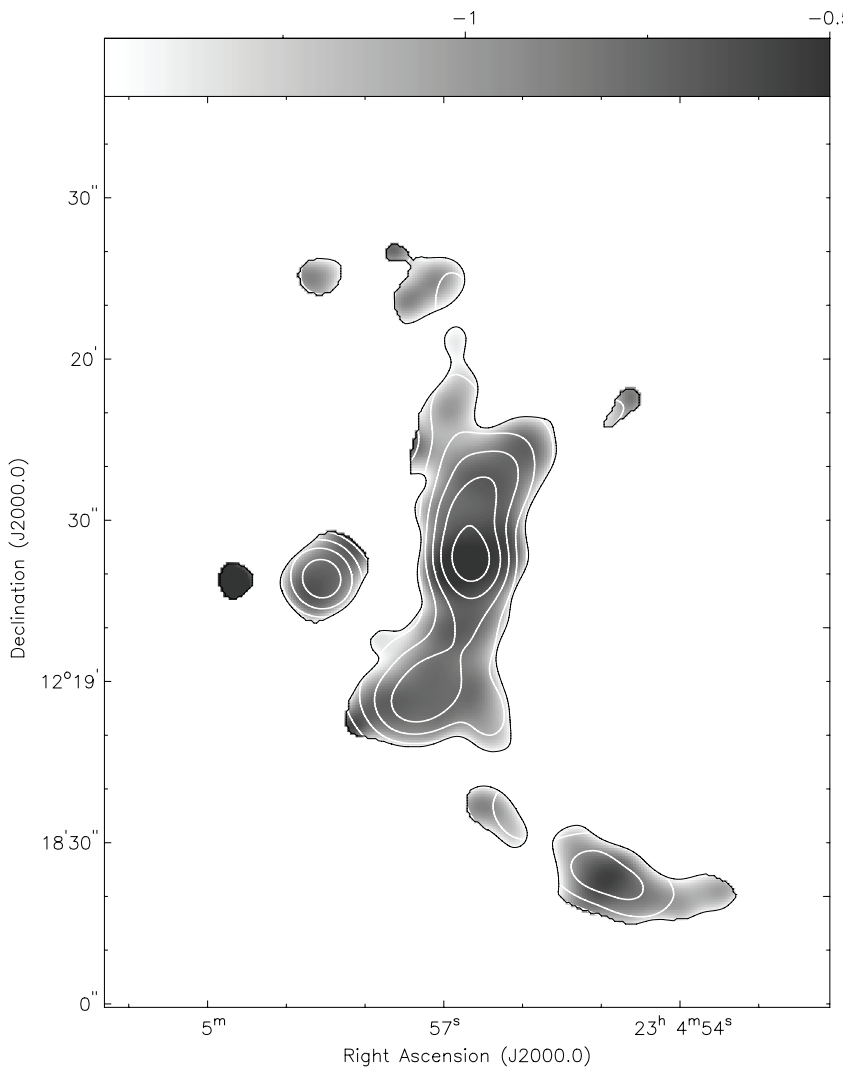

FIG. 3.-Gray-scale image of the spectral index between 3.5 and $6 \mathrm{~cm}$ at $8^{\prime \prime}$ resolution, overlaid by contours of $6 \mathrm{~cm}$ total intensity. The contours are at $(2,4,8,16,32) \times 50 \mu \mathrm{Jy}_{\text {beam }}{ }^{-1}$.

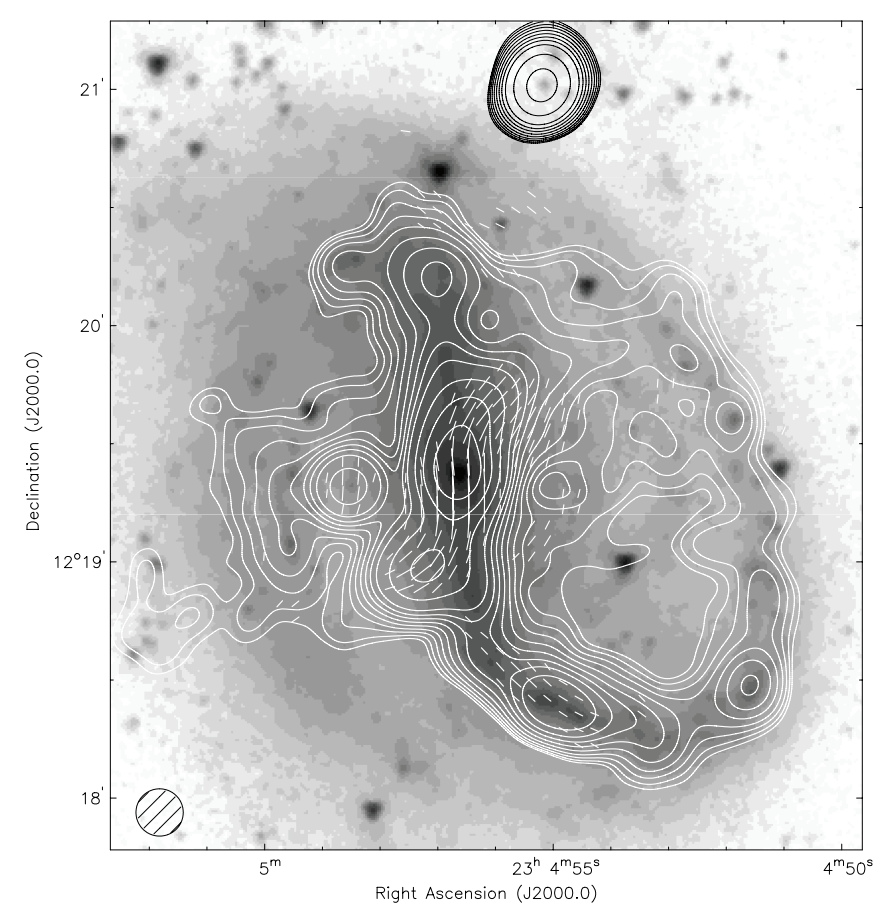

FIG. 4. $-B$-vectors $\left(E\right.$-vectors rotated by $\left.90^{\circ}\right)$ and contour image of the $3.5 \mathrm{~cm}$ total intensity at $12^{\prime \prime}$ resolution (D configuration), overlaid on an unpublished Spitzer gray-scale image of $3.6 \mu \mathrm{m}$ infrared emission at $2^{\prime \prime}$ resolution. The rms noise is $10 \mu \mathrm{Jy}_{\text {beam }}^{-1}$. The contours are at $(1,1.4,2,2.8,4,5.7,8,11,16$, $32,64,128,256) \times 20 \mu \mathrm{Jy}$ beam $^{-1}$.

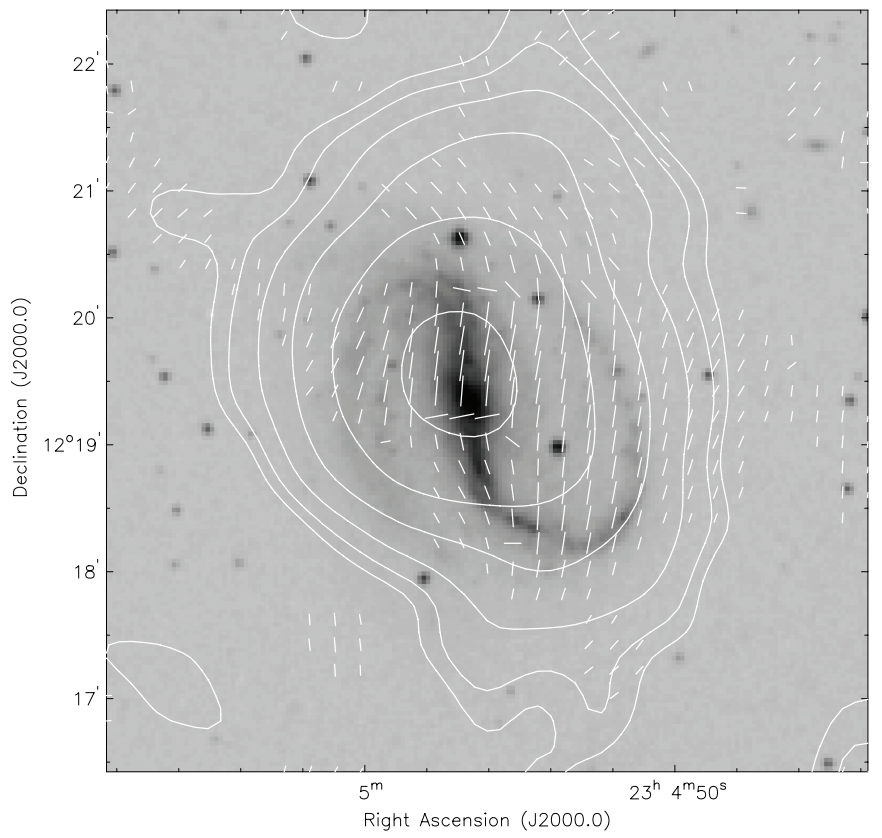

FIG. 5.-Effelsberg $B$-vectors ( $E$-vectors rotated by $90^{\circ}$ ) and contour image of the $3.6 \mathrm{~cm}$ total intensity at $90^{\prime \prime}$ resolution, overlaid on a gray-scale Digital Sky Survey optical image. The rms noise is $\simeq 400 \mu \mathrm{Jy}_{\text {beam }}{ }^{-1}$. The contours are at $(1,2,4,8,16,32) \times 400 \mu \mathrm{Jy}_{\text {beam }}{ }^{-1}$.

the nucleus are caused by the strong unpolarized emission from the nucleus. The degree of polarization at $6 \mathrm{~cm}$ is similar to that at $3.5 \mathrm{~cm}$ in the outer jet, but lower in the inner jet due to Faraday depolarization.

The $B$-vectors in Figure 6 show an ordered ${ }^{1}$ pattern and follow the jet axis. The $B$-vectors at $3.5 \mathrm{~cm}$ (Fig. $6 a$ ) are Faraday rotated by less than $10^{\circ}$ (see $\S 3.4$ ) and hence delineate the orientation of the ordered magnetic field. We conclude that the field precisely follows the $S$ shape of the jet, which is important for understanding its origin (§ 4.2).

Polarized emission is observed also outside the jet. The elongated feature at the top of Figure $6 b$ is located in the interarm region between the northern spiral arms and the northern part of the wrapping western arm and is highly polarized $(\approx 50 \%)$. Interarm polarization is typical for spiral galaxies (Beck 2005). Figure 7 shows that the interarm polarization extends to the edge of the optically visible disk of the galaxy. In contrast, the southern spiral arm shows polarization on the arm with well-aligned $B$-vectors and lower degrees of polarization of $10 \%-20 \%$ (Fig. 7). This could be due to compression as the spiral arm is sweeping the ISM of the galaxy, as suggested by the merger models of Laine \& Heller (1999). The western continuation of the arm and the bar itself do not show any significant polarized emission.

The diffuse clouds of total emission west and east of the bar (Fig. 4) are polarized at $3.5 \mathrm{~cm}$ with a well-ordered magnetic field oriented parallel to the jet axis (Fig. 7). These features are also seen in the $6 \mathrm{~cm}$ polarization map at $30^{\prime \prime}$ resolution (Beck et al. 2002). Even with the low angular resolution of our Effelsberg map

\footnotetext{
${ }^{1}$ Note that magnetic fields observed in linearly polarized emission can be either coherent (i.e., preserving their direction within the telescope beam) or incoherent (i.e., with multiple field reversals within the beam). To distinguish between these two components, additional Faraday rotation data are needed. For the sake of simplicity, the fields observed in polarization are called "ordered" throughout this paper if Faraday rotation is not measured. If Faraday rotation is measured and it is significant, "regular field" is used.
} 


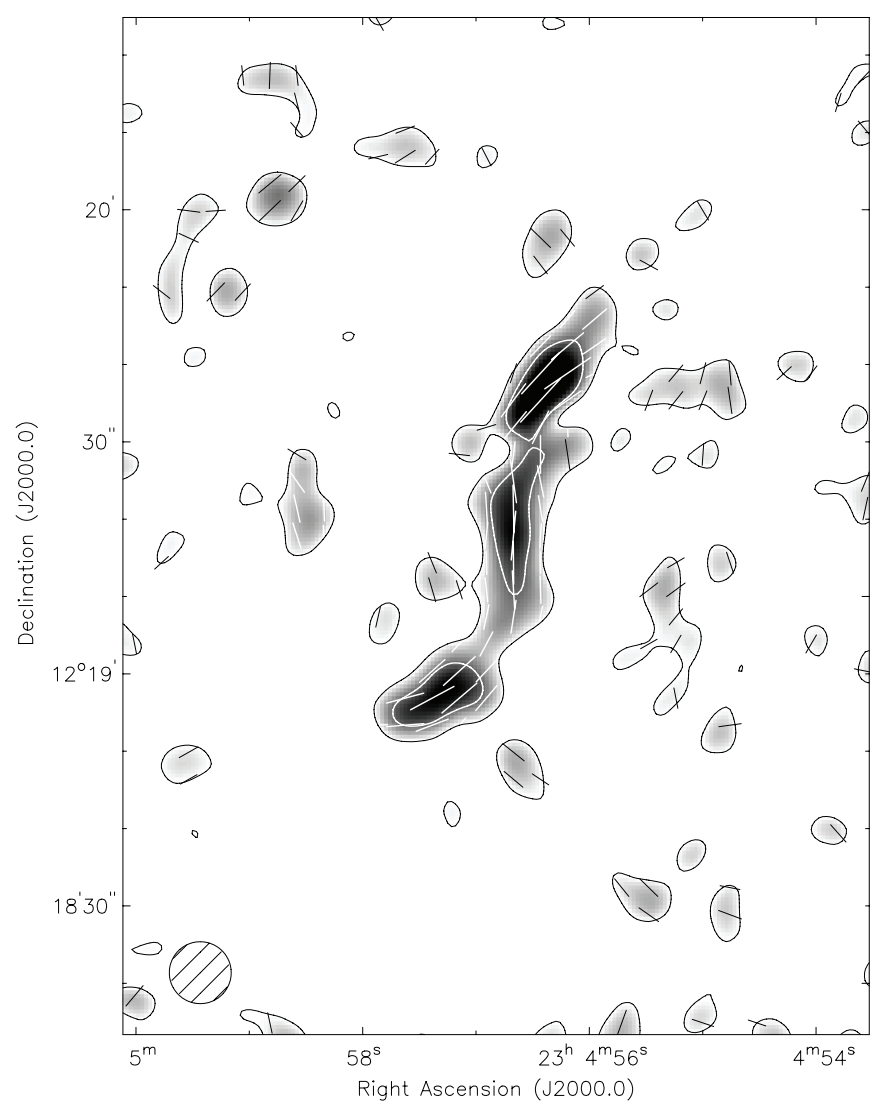

FIG. $6 a$

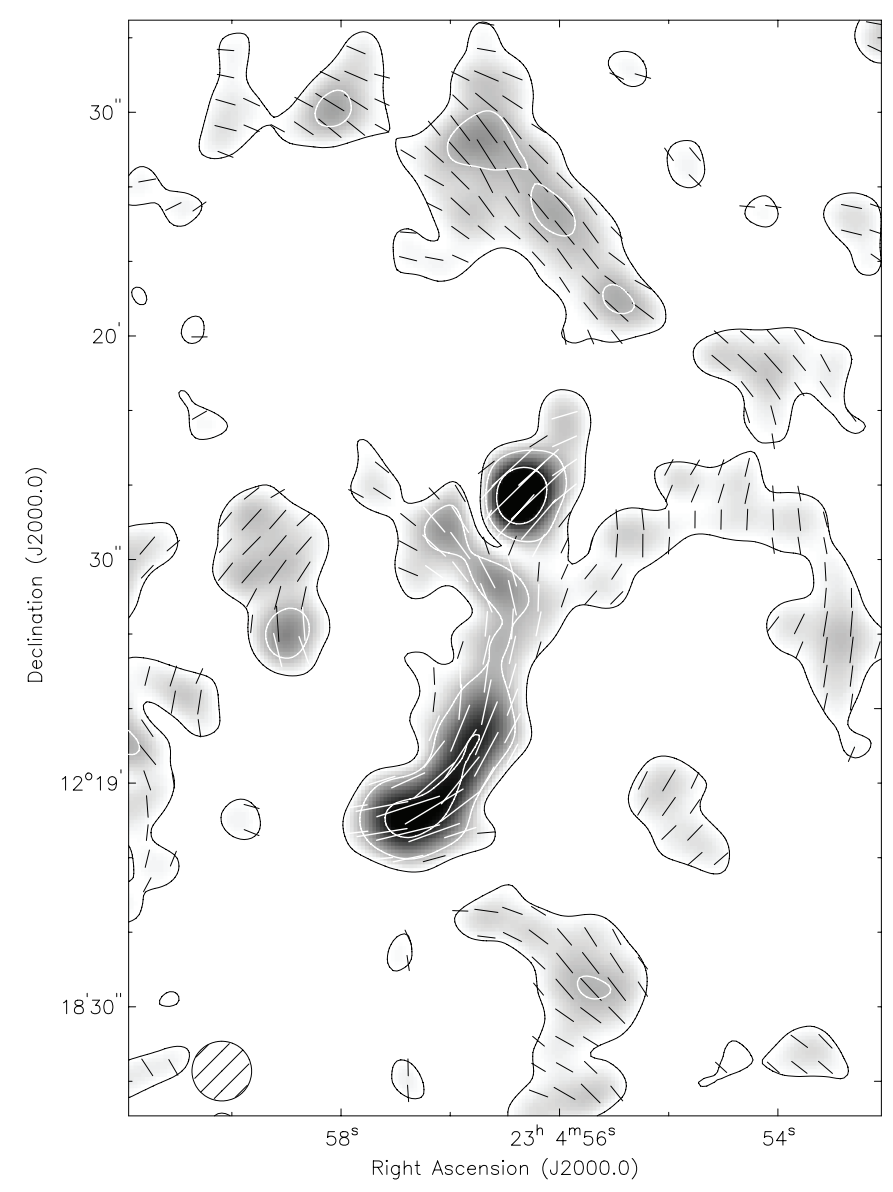

FIG. $6 b$

FIG. 6.- (a) $B$-vectors ( $E$-vectors rotated by $90^{\circ}$ ) overlaid on a contour and gray-scale image of polarized intensity of the 3.5 cm radio continuum emission at

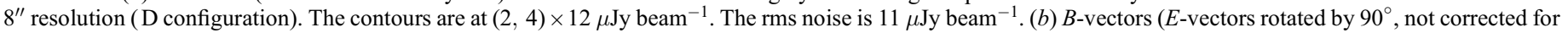

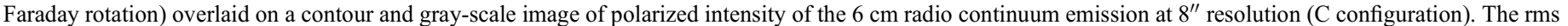
noise is $9 \mu \mathrm{Jy}_{\text {beam }^{-1}}$. The contours are at $(2,4,8) \times 10 \mu \mathrm{Jy}$ beam ${ }^{-1}$. Beams are shown by the hatched circle near the bottom left corners.

(Fig. 5), in which depolarization within the beam is strong, these regions are still weakly polarized, with similar field orientations as in Figure 7.

At 18 and $22 \mathrm{~cm}$ (Fig. 8) polarized emission is detected from the southern jet only (with $p \simeq 5 \%$ fractional polarization), while the northern jet is completely depolarized. Such asymmetric depolarization is well known in radio galaxies as the LaingGarrington effect (Garrington et al. 1988) and suggests that the northern jet is located behind the galaxy disk, which depolarizes the emission, while the southern jet is in front of the disk ( $\S 3.5)$.

The diffuse clouds east and west of the bar are polarized at $22 \mathrm{~cm}$ by $p \simeq 10 \%$. They are hardly detected at $18 \mathrm{~cm}$ because the signal-to-noise ratio is too low. Field strengths and ionized gas density are low in the outer disk, so that Faraday depolarization is smaller than in the inner parts. The overall distribution of diffuse polarized intensity at $22 \mathrm{~cm}$ is asymmetric along the major axis of the inclined disk (position angle of $22^{\circ}$ ). This phenomenon of asymmetric Faraday depolarization around $20 \mathrm{~cm}$ wavelength is observed in many spiral galaxies, e.g., in NGC 6946 (Beck 2007).

\subsection{Magnetic Field Strengths in the Disk and the Jet}

The strengths of the total magnetic field $B_{t}$ and its ordered component in the sky plane can be derived from the total synchrotron intensity and its degree of linear polarization at $8^{\prime \prime}$ resolution
(§ 3.2), assuming equipartition between the energy densities of the magnetic field and the total cosmic rays, a value for the ratio $R$ between the number densities of cosmic-ray protons and electrons, the path length $L$ through the synchrotron-emitting medium, and the synchrotron spectral index $\alpha_{n}$ (Beck \& Krause 2005). The equipartition strengths of the ordered field, either regular or incoherent, are always lower limits due to the limited resolution (beam depolarization) and Faraday depolarization effects.

The total field strength $B_{t}$ in the southern spiral arm (assuming $R=100, L=1 \mathrm{kpc}$ for the full-disk thickness, and $\alpha_{n}=$ -1.0 ; see Fig. 3 ) is $\simeq 18 \mu \mathrm{G}$, and that of the ordered field in the sky plane (not corrected for inclination) is $\simeq 7 \mu \mathrm{G}$. The strength of the random field $B_{r}$ is $\simeq 17 \mu \mathrm{G}$. Outside the bar and spiral arms the average total field strength is $\simeq 11 \mu \mathrm{G}$ and that of the ordered field in the sky plane is $\simeq 8 \mu \mathrm{G}$. The strength of the random field $B_{r}$ is $\simeq 7 \mu \mathrm{G}$. Such values are typical for regions between spiral arms in galaxies (Beck 2005).

The total equipartition field strength in the jet, assuming $R_{1}=100$ (a relativistic proton-electron plasma), $L=250 \mathrm{pc}$ ( 3.1 ), and $\alpha_{n}=-0.8$ (Fig. 3), varies from $B_{t} \simeq 35 \mu \mathrm{G}$ in the inner jet to $\simeq 40 \mu \mathrm{G}$ in the outer jet, while the strength of the component of the ordered field in the sky plane is $\simeq 8 \mu \mathrm{G}$ and almost constant. In a $40 \mu \mathrm{G}$ magnetic field, the synchrotron lifetime of cosmic-ray electrons emitting at $6 \mathrm{~cm}$ is about $10^{6} \mathrm{yr}$. If the jet is composed of a relativistic positron-electron plasma 


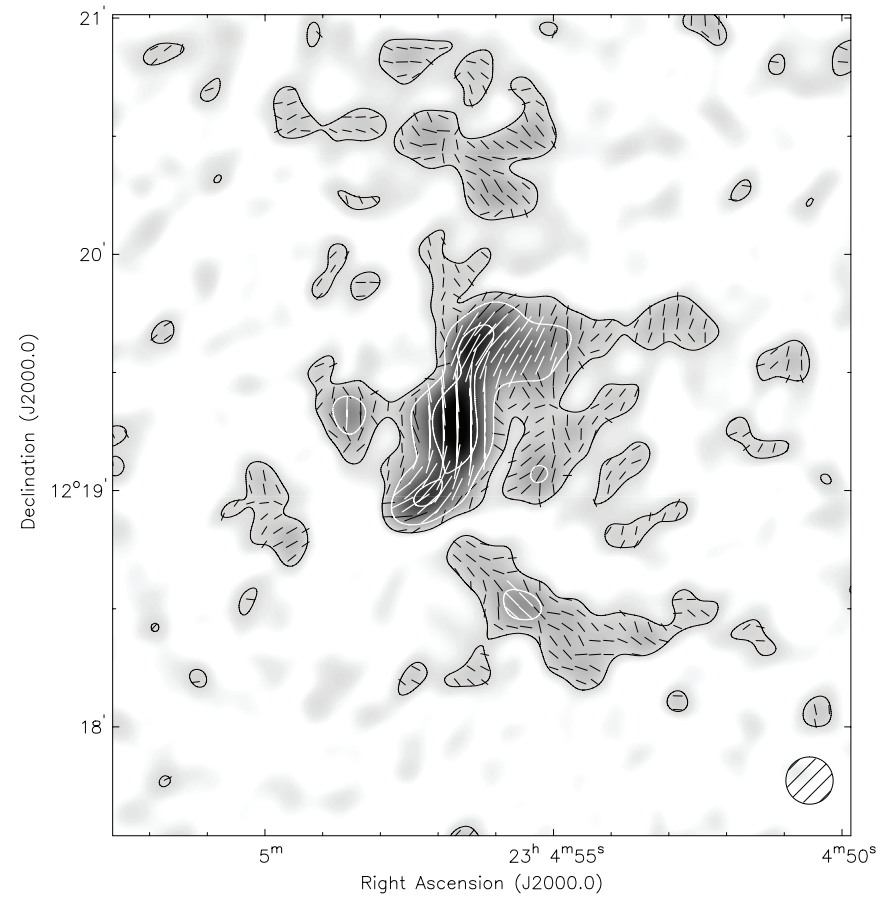

Fig. 7.- $B$-vectors $\left(E\right.$-vectors rotated by $\left.90^{\circ}\right)$ and contour image of the $3.5 \mathrm{~cm}$ polarized intensity at $12^{\prime \prime}$ resolution ( $\mathrm{D}$ configuration). The rms noise is $9 \mu \mathrm{Jy}$ beam $^{-1}$. The contours are at $(2,4,8) \times 10 \mu \mathrm{Jy}$ beam $^{-1}$.

$\left(R_{2}=0\right)$, all equipartition field strengths are smaller by a factor of $\left(R_{1}+1\right)^{1 /\left(3-\alpha_{\mathrm{n}}\right)} \simeq 3.4($ Beck \& Krause 2005$)$.

These values are based on our radio data at $8^{\prime \prime}(1.2 \mathrm{kpc})$ resolution, while the total intensity map at higher resolution (Fig. 1) reveals a width of the jet of $\simeq 250 \mathrm{pc}$, so that the internal field structure of the jet is not resolved and the polarized intensities

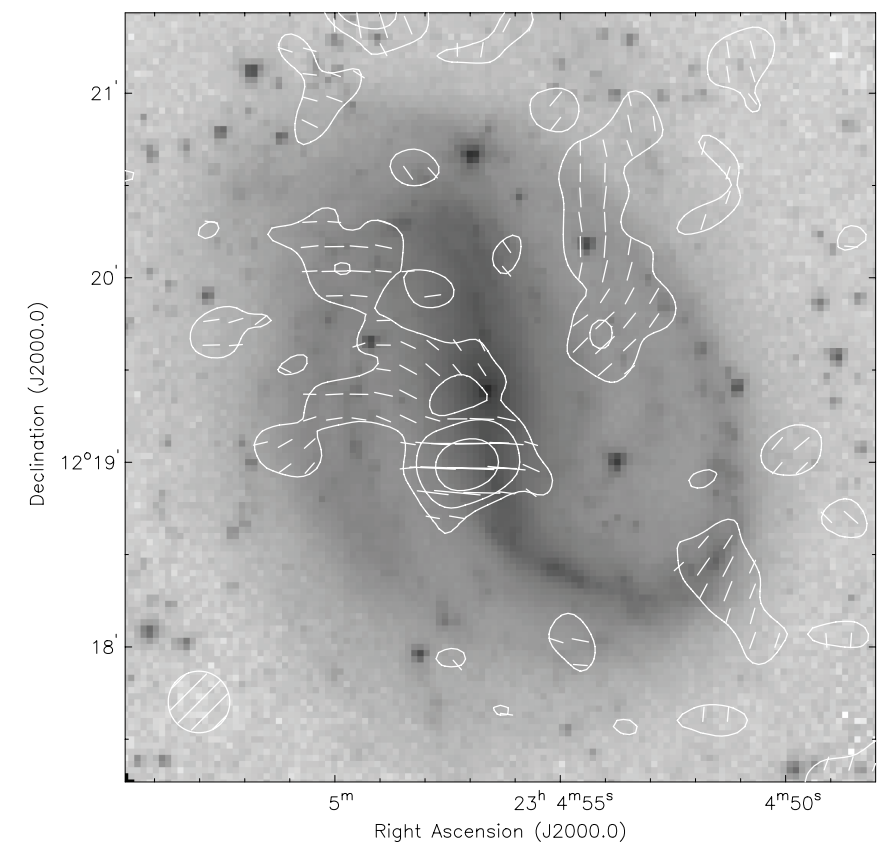

FIG. $8 a$ suffer from depolarization within the telescope beam. Hence, the equipartition value for the ordered field is a lower limit.

\subsection{Faraday Rotation: Regular Magnetic Fields}

We present the map of Faraday rotation measures (RMs) between 3.5 and $6 \mathrm{~cm}$ at $8^{\prime \prime}$ resolution in Figure 9. The RM at the location of the PI maximum in the southern spiral arm is $-60 \pm$ $20 \mathrm{rad} \mathrm{m}^{-2}$. The RM values along the jetlike feature are fluctuating between about -100 and $+300 \mathrm{rad} \mathrm{m}^{-2}$ (Fig. 10). The origin of Faraday rotation in NGC 7479 could be internal to the jet or in the ISM of the galaxy disk in front of the jet, as seen by an observer. As discussed in $\S 4.1$, the observed Faraday rotation occurs in the foreground of the jet, either in the disk of the galaxy, in a jet cocoon, or both.

The origin of Faraday rotation in the foreground disk is supported by the asymmetry in Faraday rotation, which is larger by a factor of 3-5 toward the northern jet than toward the southern jet (Fig. 9), and also by the asymmetry in the Faraday depolarization data $(\S 3.5)$. The northern jet is located behind the disk of the galaxy, while the southern jet lies in front of the disk. The jet acts as a bright polarized background, which allows us to investigate the structure of the regular magnetic field in front of the jet with unprecedented precision.

We took two RM slices, one along the inner jet and another along the outer southern jet (along the two thick lines marked in Fig. 9). The slices are shown in Figure 10. They reveal an asymmetry in the RM values along the inner jet (Fig. 10a). RM is negative in the south (i.e., the regular field points away from the observer) and positive in the north (i.e., the regular field points toward the observer). The sharp decrease of RM values at $x=30^{\prime \prime}$ is due to the strongly polarized outer northern jet, which shows small Faraday rotation. The field component responsible for the strong Faraday rotation of the inner jet is probably located in the bar dust lane, whereas the field in front of the outer jet belongs to

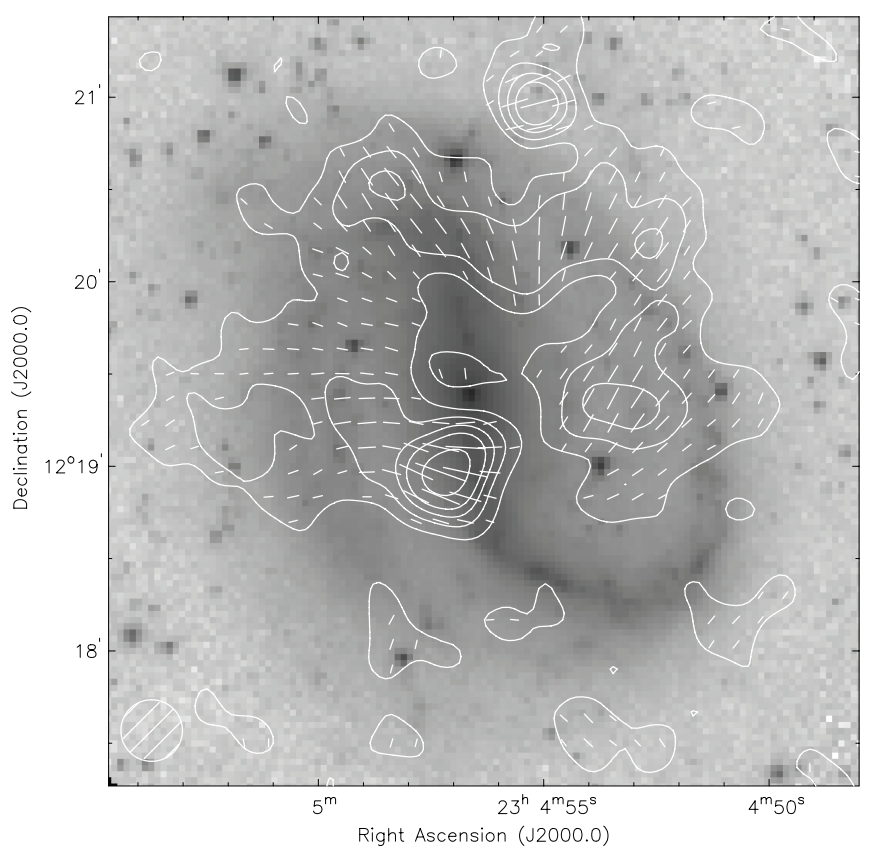

FIG. $8 b$

FIG. 8. - (a) $B$-vectors ( $E$-vectors rotated by $90^{\circ}$, not corrected for Faraday rotation) and a contour image of polarized intensity of the radio continuum emission at $18 \mathrm{~cm}$ and $(b)$ at $22 \mathrm{~cm}$ overlaid on a gray-scale Digital Sky Survey optical image, both at $20^{\prime \prime}$ resolution. The rms noises are 40 and $20 \mu \mathrm{Jy}_{\text {beam }}^{-1}$, respectively. The contours are at $(2,4,8) \times 40 \mu \mathrm{Jy} \mathrm{beam}^{-1}$ in $(a)$ and $(1,2,3,4,6) \times 45 \mu \mathrm{Jy}_{\text {beam }}^{-1}$ in $(b)$. 


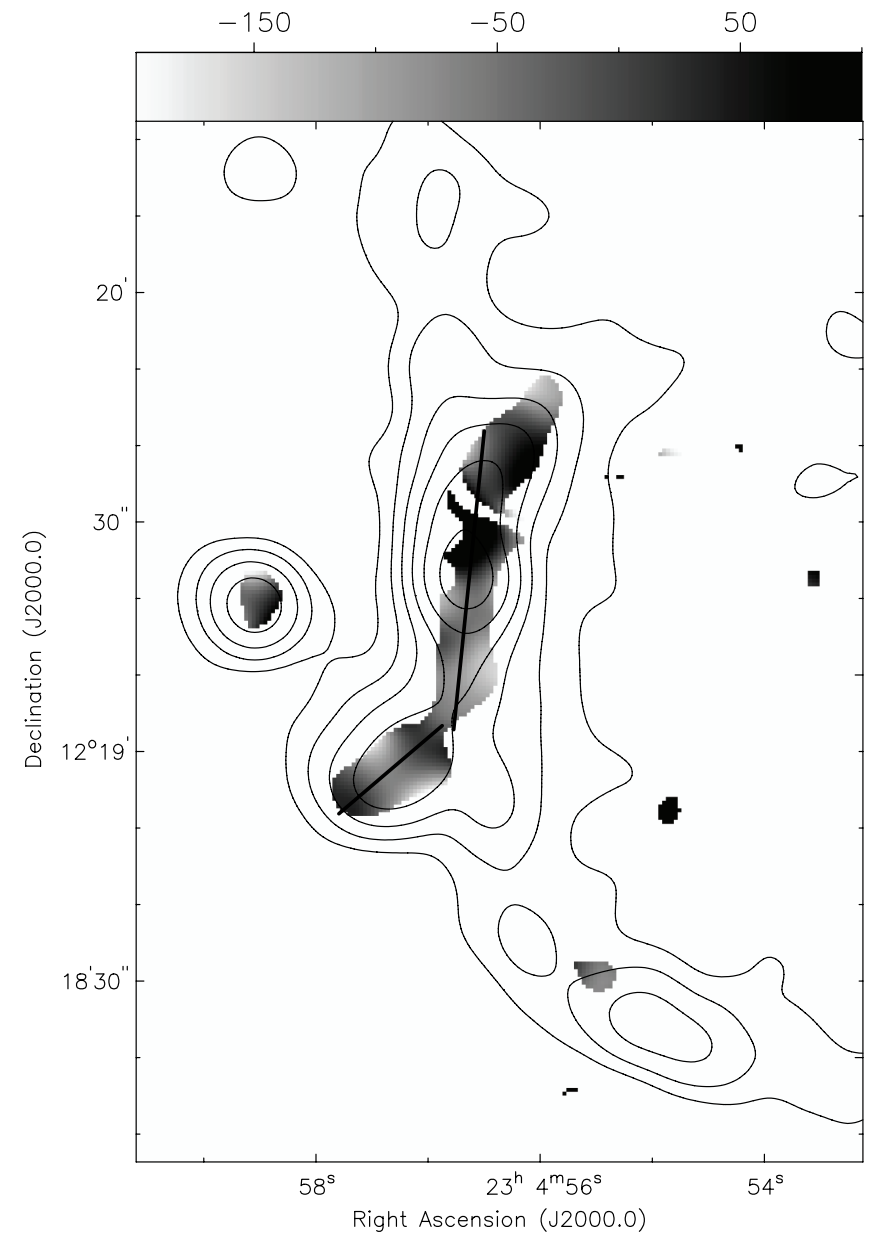

FIG. 9.-RM map between 3.5 and $6 \mathrm{~cm}$ of NGC 7479 at $8^{\prime \prime}$ resolution in gray scale, overlaid by contours of total $6 \mathrm{~cm}$ intensity. The contour levels are at $(2,4,8,16,32,64) \times 50 \mu \mathrm{Jy}_{\text {beam }}^{-1}$. The locations of the slices taken along the inner jet and the outer southern jet (Fig. 10) are indicated by thick black lines.

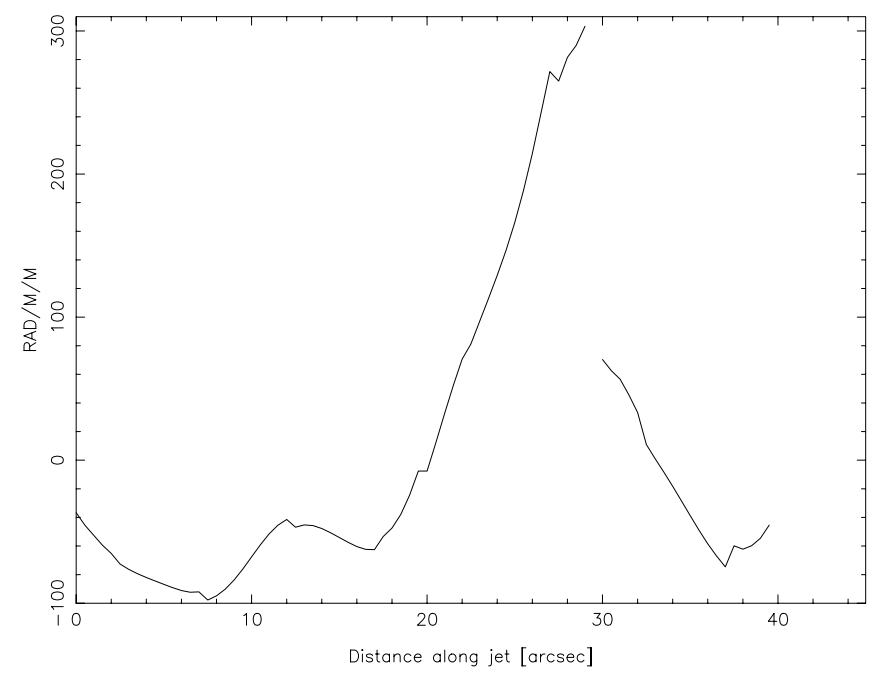

FIG. $10 a$ the diffuse disk of the galaxy and has a reversed direction with respect to the field in the bar.

The north-south RM asymmetry of the inner jet means that the regular magnetic field in the foreground, probably in the bar dust lanes, has opposite directions on the opposites sides of the nucleus. Assuming trailing spiral arms, the signs of radial velocities from H I observations (Laine \& Gottesman 1998) show that the northwestern side is the near side of the disk, so that the large-scale regular field in the dust lanes points toward the nucleus on opposite sides. The same result was derived for the barred galaxy NGC 1097 ( Beck et al. 2005). Spiral galaxies also seem to prefer inward-directed regular fields, which is a challenge for theories of magnetic field generation in galaxies (Krause \& Beck 1998).

The 6 and $20 \mathrm{~cm}$ observations of Ho \& Ulvestad (2001) with $2.5^{\prime \prime}$ resolution show a few magnetic field vectors at the northern edge of the inner jet ( $\simeq 10^{\prime \prime}$ north of the nucleus) oriented along the inner jet at both wavelengths, so that the RM seems to be small. This is inconsistent with our results, as we measure $\mathrm{RM} \simeq 300 \mathrm{rad} \mathrm{m}^{-2}$ in this region between 3.5 and $6 \mathrm{~cm}$ (Fig. 10a). The reason for this discrepancy is probably strong depolarization at $20 \mathrm{~cm}$ (see $\S 3.5)$ and hence a large uncertainty in the polarization angles in Ho \& Ulvestad (2001).

The outer southern jet (Fig. 10b) reveals variations in RM over about $20^{\prime \prime}\left(\simeq 3 \mathrm{kpc}\right.$ ) with sign reversals located at $x=6^{\prime \prime}$ and $19^{\prime \prime}$, indicating that the line-of-sight component of the regular disk field in front of this region shows two reversals on this scale. At the present resolution of $8^{\prime \prime}$ we cannot decide whether these reversals are smooth or abrupt. Two more sign reversals occur between the inner and outer southern jet (compare the first point at $0^{\prime \prime}$ in Fig. $10 a$ with the last point at $25^{\prime \prime}$ in Fig. $10 b$ ) and, on a scale of about $1 \mathrm{kpc}$, also between the inner and outer northern jet (Fig. 10a). Further reversals may occur on scales not resolved by our observations.

We show an RM map at $20^{\prime \prime}$ resolution between 3.5 and $22 \mathrm{~cm}$ in Figure 11. RM is $-30 \pm 3 \mathrm{rad} \mathrm{m}^{-2}$ in the southeast where the outer southern jet is located, about half the RM between 3.5 and $6 \mathrm{~cm}$, but of the same sign. Smaller RM values at longer wavelengths are commonly seen in galaxies and indicate Faraday

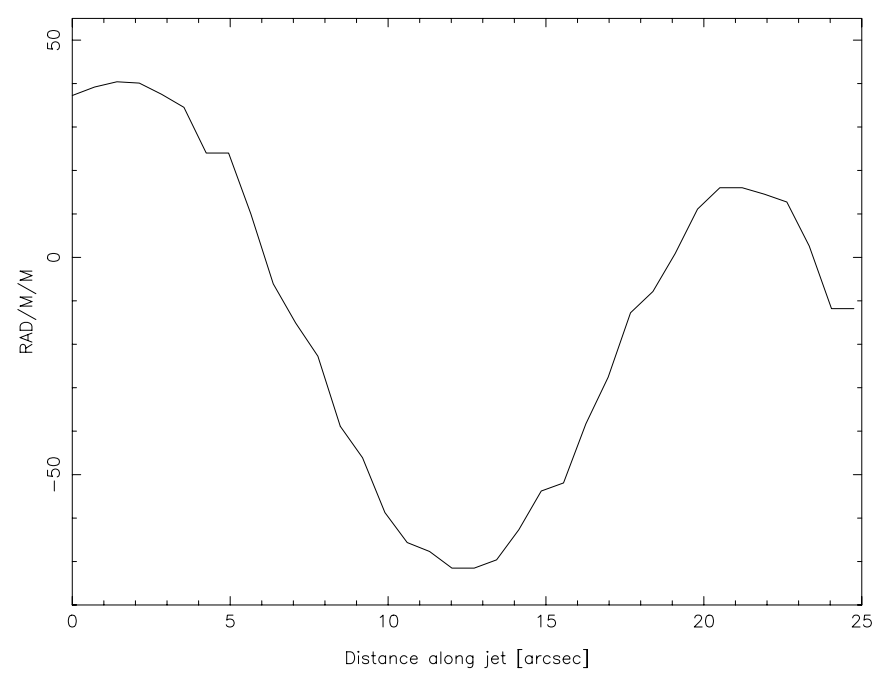

FIG. $10 b$

FIG. 10.- (a) Slice in the RM map of Fig. 9 at $8^{\prime \prime}$ resolution along the inner jet (position angle $-6^{\circ}$ ). The southernmost point is to the left. The point closest to the nucleus is located at around $20^{\prime \prime}$. (b) RM slice along the outer southern jet (position angle $-40.4^{\circ}$ ). The point at the southeastern jet end is to the left. The foreground RM in our Galaxy is estimated to be $-6 \pm 4 \mathrm{rad} \mathrm{m}^{-2}$. 


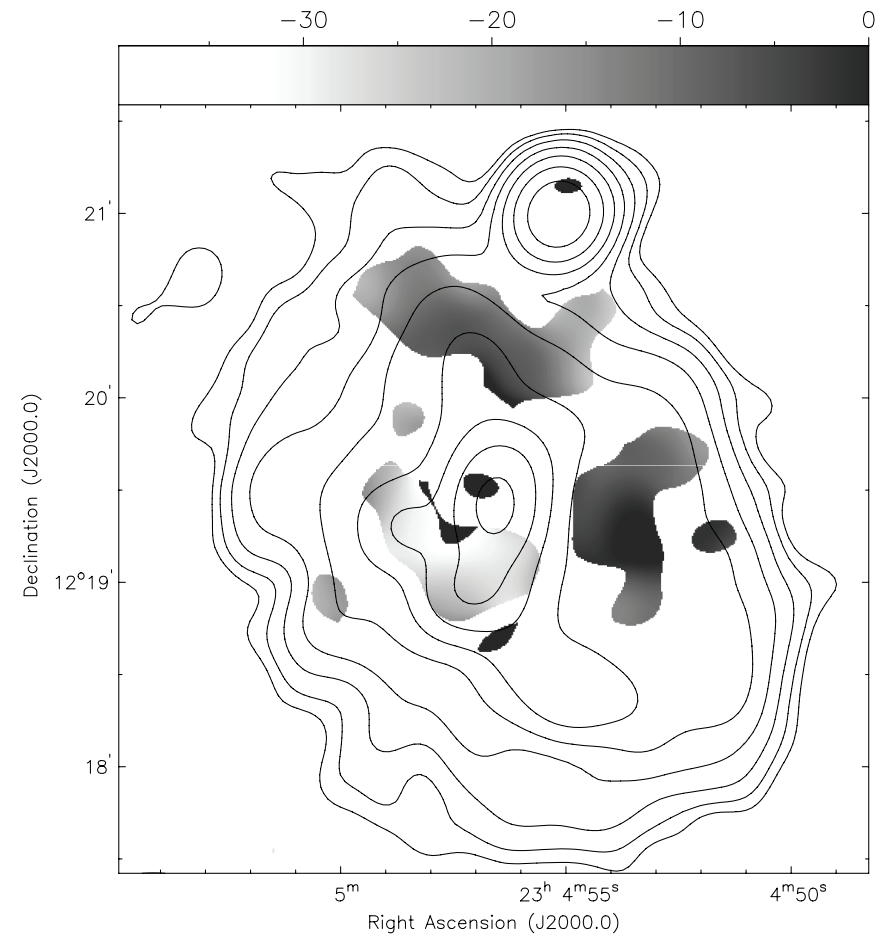

FIG. 11.-RM map between 3.5 and $22 \mathrm{~cm}$ at $20^{\prime \prime}$ resolution in gray scale, overlaid by $22 \mathrm{~cm}$ total intensity contours. The contour levels are at $(2,4,8,16,32$, $64,128,256,512) \times 30 \mu \mathrm{Jy}_{\text {beam }^{-1}}$

depolarization effects that strongly increase with wavelength (Sokoloff et al. 1998).

Both sources north of the galaxy disk (at R.A.J2000.0 = $23^{\mathrm{h}} 04^{\mathrm{m}} 55.2^{\mathrm{s}}$, decl.J2000.0 $=12^{\circ} 21^{\prime} 01^{\prime \prime}$, seen as an elongated source at $12^{\prime \prime}$ resolution in Fig. 4) have RM values of $-180 \pm$ $25 \mathrm{rad} \mathrm{m}^{-2}$. The similar RM excludes an origin internal to these sources. Their position suggests that these are background sources, unrelated to NGC 7479, and that the RM emerges in the disk of NGC 7479 and/or in the foreground of the Milky Way. The negative RM means that the regular magnetic field points away from the observer.

The polarized pointlike source about $30^{\prime \prime}$ east of the nucleus (at R.A.J2000.0 $=23^{\mathrm{h}} 04^{\mathrm{m}} 58.5^{\mathrm{s}}$, decl. ${ }_{\mathrm{J} 2000.0}=12^{\circ} 19^{\prime} 19^{\prime \prime}$ ) has an $\mathrm{RM}$ of $-20 \pm 20 \mathrm{rad} \mathrm{m}^{-2}$ that is substantially different from the northern sources. The lack of an optical or IR counterpart indicates that this is another background source. Assuming the same foreground $\mathrm{RM}$ of $\simeq-180 \mathrm{rad} \mathrm{m}^{-2}$ as in the north, the measured $\mathrm{RM} \simeq-20 \mathrm{rad} \mathrm{m}^{-2}$ would require an exceptionally large internal $\mathrm{RM}_{i}$ of $\simeq+160 \mathrm{rad} \mathrm{m}^{-2}$. More likely, the regular magnetic field in the disk of NGC 7479 and/or in the foreground is reversed and points toward the observer in this region. A more detailed investigation requires separation of the component of Faraday rotation $\mathrm{RM}_{\mathrm{fg}}$ occurring in the foreground of the Milky Way.

As an estimate of $\mathrm{RM}_{\mathrm{fg}}$ in the direction to NGC 7479 we use the average value of RM between 3.5 and $22 \mathrm{~cm}$ in the western and northern regions, far outside the jet, which is $-6 \pm 4 \mathrm{rad} \mathrm{m}^{-2}$ (Fig. 11). The RM sky, interpolated from the RM grid of polarized extragalactic sources (Johnston-Hollitt et al. 2004), also yields values of around $0 \mathrm{rad} \mathrm{m}^{-2}$ at the position of NGC 7479 in Galactic coordinates ( Table 1). The RM sky shown by Han in Wielebinski (2005) gives small negative values.

Knowing $\mathrm{RM}_{\mathrm{fg}}$, we can investigate the regular magnetic field in the disk of NGC 7479 in some more detail. The observed RM of a background source corresponds to an average $\mathrm{RM}_{0}$ through the disk of $\mathrm{NGC} 7479$ of $\mathrm{RM}_{0}=\left(\mathrm{RM}-\mathrm{RM}_{\mathrm{fg}}-\mathrm{RM}_{i}\right) / 2$, while $\mathrm{RM}_{0}=\mathrm{RM}-\mathrm{RM}_{\mathrm{fg}}$ is valid for diffuse polarized emission from the galaxy itself (if Faraday depolarization is small). The two northern background sources (neglecting internal rotation $\mathrm{RM}_{i}$ ) give $\mathrm{RM}_{0}=-87 \pm 13 \mathrm{rad} \mathrm{m}^{-2}$, which agrees (within the uncertainties) with $\mathrm{RM}_{0}=-54 \pm 20 \mathrm{rad} \mathrm{m}^{-2}$ of the diffuse polarized emission in the southern spiral arm, so that the strength and direction of the regular field are similar in the outer northern disk and in the southern spiral arm. However, the polarized source in the east with $\mathrm{RM}_{0}=-7 \pm 10 \mathrm{rad} \mathrm{m}^{-2}$ and the multiple reversals in front of the jet (see above) show that the large-scale magnetic field cannot have a simple symmetry as observed in several spiral galaxies (Beck 2005).

Strong variations and frequent reversals of the regular field in a galaxy disk are predicted for a mean field dynamo with a long timescale (Beck et al. 1994). Massive bars lead to nonaxisymmetric gas flows that affect dynamo action (Moss et al. 2007). Furthermore, shearing flows around bars or spiral arms can generate field loops with strong polarized emission but frequent field reversals on scales smaller than the beam size (Beck et al. 2005). The detection of such reversals is generally difficult because the polarized intensities of normal barred galaxies are low. RM reversals on $\approx 1 \mathrm{kpc}$ scale were found in the massive spiral galaxy NGC 6946 (Beck 2007) but are hardly significant. NGC 7479 with its bright jet is so far the most promising laboratory for further studies at higher resolution.

\subsection{Faraday Depolarization: Turbulent Magnetic Fields}

Wavelength-dependent Faraday depolarization (DP) is a powerful tool to detect ionized gas embedded in regular or turbulent magnetic fields (Sokoloff et al. 1998). DP data are crucial for an investigation of the origin of Faraday rotation: DP may be caused by internal differential Faraday rotation in the regular magnetic fields within the synchrotron-emitting medium, by internal Faraday dispersion by turbulent fields within the emitting medium, or by external Faraday dispersion by turbulent fields in the foreground medium. In the first case, DP and $|\mathrm{RM}|$ are anticorrelated; otherwise, they are independent.

Faraday depolarization is usually defined as the ratio DP of the degrees of polarization of the synchrotron emission at two wavelengths. This requires subtraction of the thermal emission, which is unreliable with data at only two radio frequencies. Instead, $\mathrm{DP}$ in this paper was computed by $\mathrm{DP}=\left(\mathrm{PI}_{1} / \mathrm{PI}_{2}\right)\left(\nu_{2} / \nu_{1}\right)^{\alpha_{n}}$, where $\alpha_{n}=-1.0$ is the synchrotron spectral index, assumed to be constant across the galaxy. Deviations in $\alpha_{n}$ affect DP less severely than the uncertainty of thermal fraction.

Figures 12 and 13 show DP values between 3.5 and $6 \mathrm{~cm}$ and between 6 and $22 \mathrm{~cm}$, respectively. DP along the jet (Fig. 12) varies between 0.6 and 1 and shows fluctuations that are not correlated or anticorrelated with those of RM (Fig. 10). Thus, Faraday rotation and depolarization must occur in front of the jet.

The asymmetric DP between 6 and $22 \mathrm{~cm}$ in the jet of NGC 7479 (Fig. 13) is an indication that the northern jet lies behind the galaxy disk and the polarized emission at 18 and $22 \mathrm{~cm}$ is depolarized by the ISM in the disk. H I absorption measurements could reveal more clearly which side of the jetlike feature is behind the disk. Unfortunately, the observations of Laine \& Gottesman (1998) revealed little neutral atomic H I gas within the bar radius of the nucleus.

Internal RM dispersion along the line of sight can be described as $\sigma_{\mathrm{RM}}=0.81 n_{e} B_{r} d(L f / d)^{0.5}$ (Sokoloff et al. 1998), where $n_{e}$ is the thermal electron density (in $\mathrm{cm}^{-3}$ ), $B_{r}$ is the isotropic random field strength (in $\mu \mathrm{G}$ ), $L$ is the path length through the thermal 


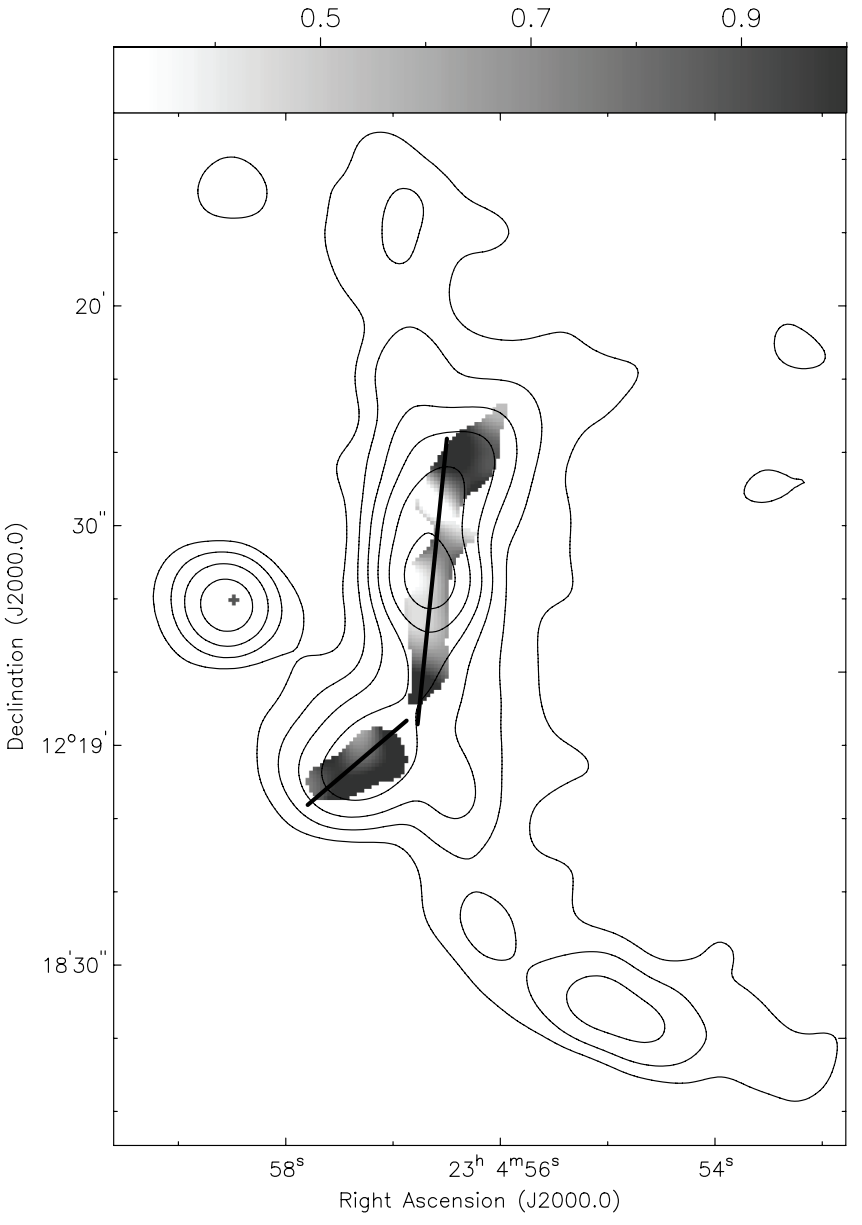

FIG. 12.-Depolarization map between 3.5 and $6 \mathrm{~cm}$ at $8^{\prime \prime}$ resolution in gray scale, overlaid by contours of $6 \mathrm{~cm}$ total intensity. The contour levels are at $(2,4,8,16,32,64) \times 50 \mu \mathrm{Jy}_{\text {beam }}{ }^{-1}$. The locations of the slices taken along the inner jet and the outer southern jet (Fig. 14) are indicated by thick black lines.

gas (in pc), $d$ is the turbulent scale (in pc), and $f$ is the filling factor of the diffuse Faraday-rotating gas. Depolarization occurs if the number of turbulent cells $N$ along the line of sight is large $(N=L f / d \gg 1)$. Then the foreground depolarization is DP = $e^{-2 \sigma_{\mathrm{RM}}^{2} \lambda^{4}}$ (Sokoloff et al. 1998). Values of $n_{e}=0.03 \mathrm{~cm}^{-3}$ outside the bar ( $\S 4.1), B_{r} \simeq 7 \mu \mathrm{G}(\S 3.3)$, and standard ISM values of $L=500 \mathrm{pc} / \cos i, d=50 \mathrm{pc}$, and $f=0.5$ give $\sigma_{\mathrm{RM}} \simeq$ $24 \mathrm{rad} \mathrm{m}^{-2}$. This yields negligible depolarization at $3.5 \mathrm{~cm}$ $(\mathrm{DP} \simeq 0.998)$ and at $6 \mathrm{~cm}(\mathrm{DP} \simeq 0.98)$, consistent with the data in the outer southern jet (Fig. 14b), and strong depolarization at $22 \mathrm{~cm}(\mathrm{DP} \simeq 0.05)$, which explains the total depolarization of the northern jet at 18 and $22 \mathrm{~cm}$ (Fig. 8). In the outer southern jet, which is bent above the disk as seen by an observer, assuming $L \simeq 250 \mathrm{pc} / \cos i$ yields $\sigma_{\mathrm{RM}} \simeq 17 \mathrm{rad} \mathrm{m}^{-2}$ and $\mathrm{DP} \simeq 0.26$ at $22 \mathrm{~cm}$, leaving some polarized emission at this wavelength. However, the condition $L f / d \gg 1$ is not fulfilled here so that depolarization will be small ( $\mathrm{DP} \simeq 1$ ), as observed (Fig. 13).

In the bar, we use the same random field strength as in the southern spiral arm of $B_{r} \simeq 17 \mu \mathrm{G}$ because the radio intensities are similar in the bar and in the southern spiral arm, and $n_{e} \simeq$ $0.06 \mathrm{~cm}^{-3}$ as derived for the bar in $\S 4.1$. This yields $\sigma_{\mathrm{RM}} \simeq$ $120 \mathrm{rad} \mathrm{m}^{-2}$ and hence significant depolarization $(\mathrm{DP} \simeq 0.69$ ) of the inner jet at $6 \mathrm{~cm}$, as observed (Fig. 14a). In summary, our simple model of Faraday dispersion by cells of random fields can explain the depolarization data in NGC 7479.

Ho \& Ulvestad (2001) appear to have detected polarized emission at $20 \mathrm{~cm}$ at $2.5^{\prime \prime}$ resolution at both ends of the inner jet

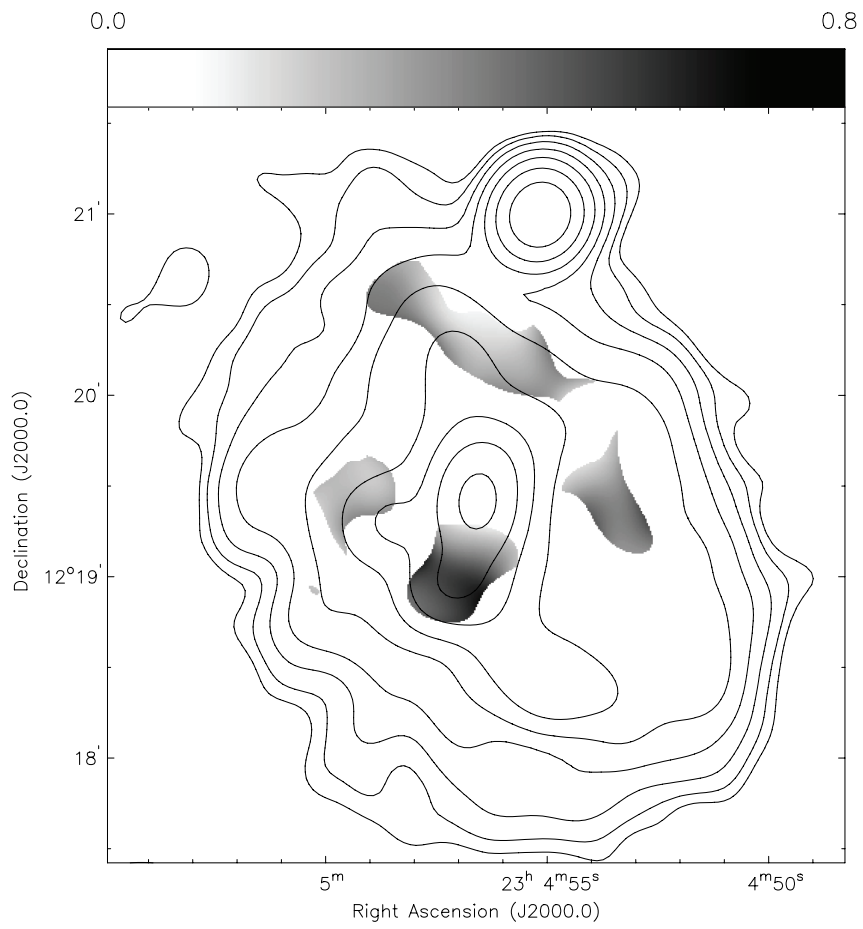

FIG. 13.-Depolarization map between 6 and $22 \mathrm{~cm}$ at $20^{\prime \prime}$ resolution in gray scale, overlaid by contours of $22 \mathrm{~cm}$ total intensity. The contour levels are at $(2,4,8,16,32,64,128,256,512) \times 35 \mu \mathrm{Jy} \mathrm{beam}^{-1}$.

( $\simeq 10^{\prime \prime}$ north and south from the nucleus), while we do not detect any polarized emission from the inner jet at 18 and $22 \mathrm{~cm}$ at $20^{\prime \prime}$ resolution (Fig. 8). Faraday dispersion in the foreground disk could be smaller than in our observations if some of the random field is resolved by the beam of Ho \& Ulvestad (2001). However, the resolution of their maps of about $400 \mathrm{pc}$ at the distance of NGC 7479 is still much larger than the typical turbulence scale of the random field. One may also consider the possibility that the brightest ridge of polarized emission in the jet is smaller than the random cell size in the foreground, so that depolarization would decrease with better angular resolution. More reliable high-resolution polarization observations around $20 \mathrm{~cm}$ are needed to study the field structure of the jet and depolarization mechanisms in more detail.

\section{DISCUSSION}

\subsection{Origin of the Faraday Rotation in Front of the Jet}

The nondetection of $\mathrm{H} \alpha$ emission from warm gas in the jet sets an upper limit for the emission measure of about $5 \mathrm{pc} \mathrm{cm}-6$ (S. Vogel 1994, private communication). With a path length of 250 pc (equal to the jet width; see $\S 3.1$ ) this yields an upper limit of the mean electron density of $\left\langle n_{e}\right\rangle \leq 0.14 \sqrt{f} \mathrm{~cm}^{-3}$ in the jet, where $f$ is the volume filling factor of the ionized gas (for the diffuse gas $f \approx 0.5$ is generally assumed). A two-component fit to the X-ray emission integrated out to $30^{\prime \prime}$ radius (see Fig. 15 for an XMM-Newton X-ray image of NGC 7479 with radio continuum contours overlaid) gives a nuclear component similar to that found by Panessa et al. (2006) plus a component of hot $\left(\approx 4 \times 10^{6} \mathrm{~K}\right)$ gas with a density of $\approx 0.03 / \sqrt{f} \mathrm{~cm}^{-3}$ (again assuming a path length of $250 \mathrm{pc}$ ), which is even less than the upper limit of the warm gas. With a mean path length through the jet of $125 \mathrm{pc},\left\langle n_{e}\right\rangle \leq 0.1 \mathrm{~cm}^{-3}$ and $B_{\text {reg }} \simeq 8 \mu \mathrm{G}$ give $|\mathrm{RM}| \leq$ $80 \mathrm{rad} \mathrm{m}^{-2}$ from the jet, which is much smaller than observed in the inner northern jet (Fig. 9). 


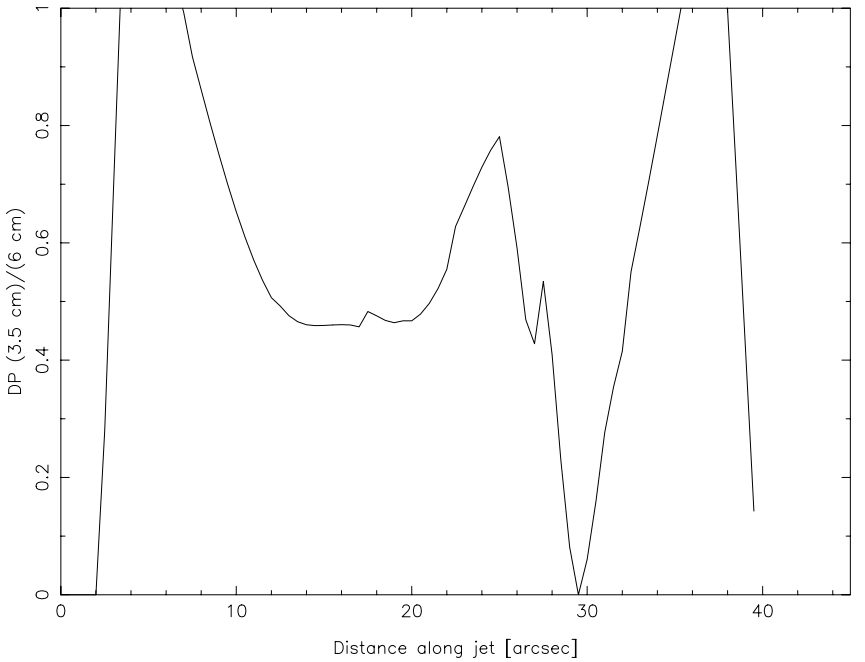

FIG. $14 a$

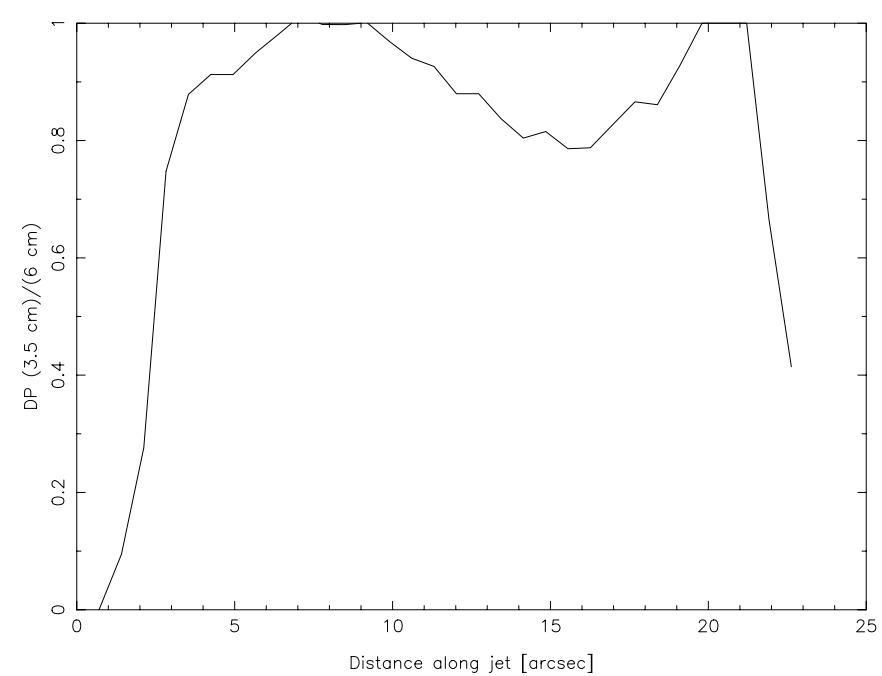

FIG. $14 b$

FIG. 14.- (a) Slice in the DP map of Fig. 12 at $8^{\prime \prime}$ resolution along the inner jet (position angle $-6^{\circ}$ ). The southernmost point is to the left. $(b)$ DP slice along the outer southern jet ( position angle $-40.4^{\circ}$ ). The point at the southeastern jet end is to the left. The positions of the slices are the same as in Fig. 10 .

Our Faraday rotation data of NGC 7479 can be explained as the effect of the magnetoionic disk of the galaxy with a full width of about $1 \mathrm{kpc}$ if the jet is located almost in the plane of the galaxy. Assuming that the ordered disk field with a component in the sky plane of $8 \mu \mathrm{G}(\S 3.3)$ has a Faraday-rotating regular component $B_{\text {reg }}$ along the line of sight of similar strength and that the effective path length of the Faraday-rotating medium is $500 \mathrm{pc} / \cos i$ (i.e., the full thickness of the disk, seen under an inclination of $51^{\circ}$ ), the observed $\mathrm{RM}$ amplitude of $\simeq 300 \mathrm{rad} \mathrm{m}^{-2}$ in front of the inner northern jet needs a thermal electron density of $0.06 \mathrm{~cm}^{-3}$, which is reasonable for the bar region with a high star formation activity. In front of the outer southern jet, RM is

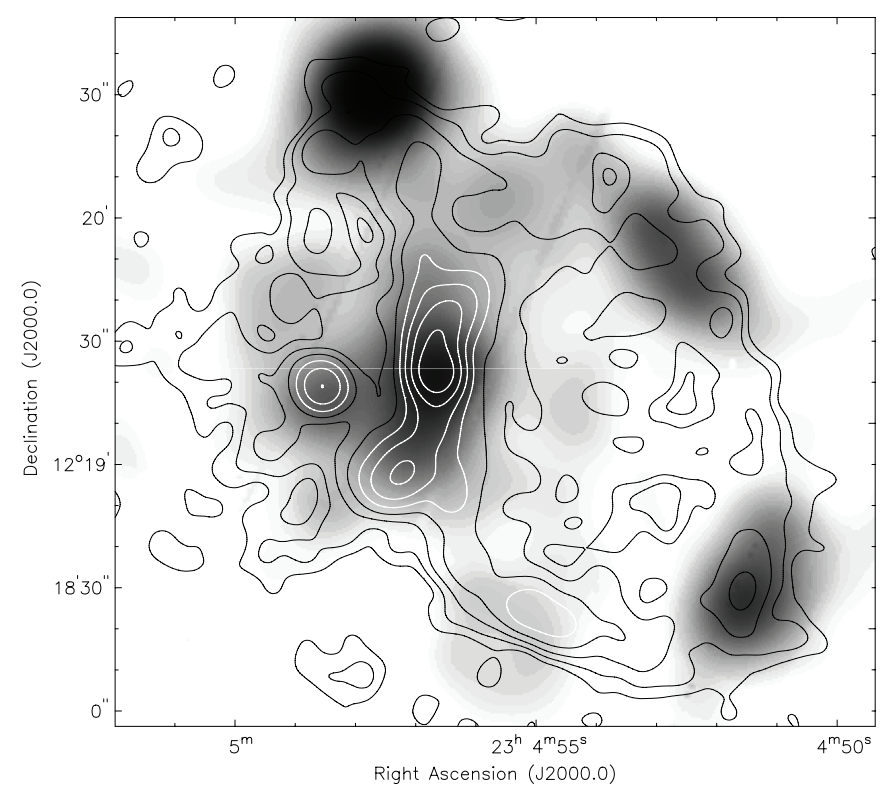

FIG. 15.-XMM-Newton X-ray image ( gray scale) in the $0.5-1.0 \mathrm{keV}$ energy band of NGC 7479. The image was made by combining information from the three EPIC cameras, pn+MOS1+MOS2, and is shown in arbitrary units (M. Ehle 2007, private communication). The image has been smoothed with a circular Gaussian beam of $15^{\prime \prime}$ FWHM. The integration time after removal of high background periods was about $6.5 \mathrm{ks}$ for pn and $9 \mathrm{ks}$ each for MOS1 and MOS2. A contour image of the $6 \mathrm{~cm}$ total intensity emission from Fig. $2 b$ is overlaid at $8^{\prime \prime}$ resolution. The contours are at $(2,4,8,16,32,64,128,256) \times 10 \mu \mathrm{Jy} \mathrm{beam}^{-1}$. significantly smaller because the jet bends out of the disk toward the observer. Assuming the same $B_{\text {reg }}$ of $8 \mu \mathrm{G}$ and an effective path length of $250 \mathrm{pc} / \mathrm{cos} i$, the observation of $|\mathrm{RM}| \simeq 70 \mathrm{rad} \mathrm{m}^{-2}$ requires a thermal electron density of $0.03 \mathrm{~cm}^{-3}$, which is typical for regions in the disks of spiral galaxies with low star formation activity.

Our Faraday depolarization data show that the jet is bent out of the plane, with the southern jet in front and the northern jet behind the plane as seen by an observer ( $\S 3.5)$. However, at a mean distance in the galaxy plane of about $6 \mathrm{kpc}$ from the center, the jet cannot be bent by more than a few degrees away from the plane; otherwise, its southern part would be located above the main part of the thermal disk, assuming a full width of $1 \mathrm{kpc}$ as is typical for spiral galaxies, so that little Faraday rotation would be observed.

Strong interaction of the jet boundaries with the ISM gas in the disk also explains the high synchrotron brightness of the jet as a compression of the existing magnetic field in the disk. If a shock exists in the boundary layer, cosmic-ray particles may also be accelerated or reaccelerated, which would further increase the synchrotron brightness of the jet. As the interstellar gas is also compressed and heated in the shock, X-ray and $\mathrm{H} \alpha$ emission is expected around the jet. More sensitive observations at these wavelengths are needed to search for such emission.

Signs of recent perturbation by a minor merger are obvious in NGC 7479, including the western spiral arm and the clouds of diffuse, polarized emission between the bar and the outer spiral arms (Figs. 5 and 8). Hence, we have to consider the possibility that the disk of ionized gas of NGC 7479 is more extended than in noninteracting systems and also most likely puffed up and strongly distorted (e.g., Walker et al. 1996). This would allow for somewhat larger angles between the jet and the disk plane.

Another source of Faraday rotation could be ionized gas in a cocoon surrounding the jet and generated by instabilities in a transonic shear layer (Bicknell et al. 1990), which would naturally explain the observed frequent field reversals as helical field loops. However, Faraday rotation of up to $300 \mathrm{rad} \mathrm{m}^{-2}$ and the nondetection of thermal emission from the jet (see above) would require regular fields along the line of sight with strengths of up to $37 \mu \mathrm{G}$ for a path length of $100 \mathrm{pc}$, much stronger than the equipartition strength of the ordered magnetic field in the jet 
itself ( $\S 3.3$ ), which seems implausible. More sensitive $\mathrm{H} \alpha$ observations are needed to search for thermal emission from such a cocoon.

The observed RM could also emerge from a combination of the galaxy disk and the jet cocoon in the northern jet that is located behind the disk, while the RM in the southern jet would be solely due to the cocoon. This scenario would allow for a large angle between the jet and the disk. However, radio lobes and hot spots are missing in NGC 7479. Without such a source of particle acceleration, the high radio brightness of an extraplanar jet is hard to explain.

\subsection{Field Structure in the Jet}

The north-south asymmetry in total intensity (intensity ratio of $q \simeq 1.3$ at 3.5 and $6 \mathrm{~cm}$ ) could be due to the viewing angle if the northern part of the jet points toward us. However, our depolarization data strongly suggest that the northern jet is located behind the galaxy disk $(\S 3.5)$ and hence points away from us. On the other hand, the fact that the southern jet is not brighter than the northern one is indication that the jet velocity cannot be relativistic.

Our polarization data show a field orientation that is almost perfectly along the jet. On the other hand, the degree of polarization is relatively low $(\S 3.2)$, so that a significant part of the field is not resolved by the telescope beam ( $8^{\prime \prime}$ or about $1.3 \mathrm{kpc}$ in most maps shown in this paper). The ratio of the unresolved field to the ordered field, projected into the sky plane, is about 3 (§3.3). Such a ratio could be explained by a helical field in the jet (Laing 1981). Radio observations with higher resolution and higher sensitivity with the EVLA are required to determine the profile of total and polarized intensity across the jet and hence the field geometry and orientation with respect to the observer.

\subsection{Bending and Triggering of the Jet}

One of the most interesting features in our radio maps is the reversed pitch angle of the magnetic field orientation along the jet compared to that of the spiral arms. Such a "leading" spiral feature can be generated by ram pressure of the ISM gas on the jet near the plane with ejection velocities of about $1000 \mathrm{~km} \mathrm{~s}^{-1}$ (Sanders 1982). On the other hand, the jet of NGC 4258 is presumably also located near the plane of its host galaxy and has the same sense of bending as the spiral arms. Ram pressure can hardly play a role for bending the jet; hence, the ejection velocities are probably larger than $1000 \mathrm{~km} \mathrm{~s}^{-1}$ in NGC 7479 (see below) and also in NGC 4258 (Daigle \& Roy 2001).

Precession of the jet opposite to the rotation of the galaxy is the most probable cause for the reversed jet bending. The precession of the jet may be driven by the precession of the accretion disk near the nucleus due to the Lense-Thirring effect ( $\mathrm{Lu}$ 1990; Merritt \& Ekers 2002), by precession of the accretion disk caused by radiation pressure (Maloney et al. 1996), or by a merger event that resulted in a binary black hole (Begelman et al. 1980). It has been suggested (Quillen et al. 1995; Laine \& Heller 1999) that NGC 7479 has recently undergone or is undergoing a minor merger event. According to Laine \& Heller (1999), the position of the merging nucleus is now at the bright near-infrared and $\mathrm{H} \alpha$ knot about $17^{\prime \prime}$ north of the nucleus.

As the jet is probably located near the plane and interacts with the ISM gas, differential rotation of the galaxy would change the orientation of the precessing jet to form a trailing spiral, opposite to what is observed in NGC 7479. For a rough estimate of this effect we take the rotation speed as $180 \mathrm{~km} \mathrm{~s}^{-1}$ at $7 \mathrm{kpc}$ radius (Laine \& Gottesman 1998). To avoid significant bending of the jet by differential rotation, the jet propagation speed has to be larger than $\simeq 5000 \mathrm{~km} \mathrm{~s}^{-1}$, which gives a travel time along the jet, and hence an age, of less than $10^{6} \mathrm{yr}$, consistent with the synchrotron age of the cosmic-ray electrons ( $\S 3.3)$.

The anomalous $15 \mathrm{kpc}$ scale radio continuum structure that we see emanating from the nucleus of NGC 7479 as a radio jet could be indirectly related to the minor merger event (perturbation of the underlying gravitational potential of the parent galaxy leading to perhaps episodic fueling of the nucleus), which may have also created the bar and the asymmetric spiral arm structure. The timescale of the minor merger, several times $10^{8} \mathrm{yr}$, is naturally much longer than the timescale of the jet (of order $10^{6} \mathrm{yr}$ ). Further evidence for recent global perturbations is seen in optical images (e.g., Fig. 1), which show anomalous dust lanes intersecting the bar at almost right angles. One such crossing point is in the bar near the southeastern jet extension. The dust lanes come from the western side of the bar, intersect the bar at almost right angles, and can be followed on the eastern side of the bar for a short distance.

It is also possible that as the jet is propagating near the plane of the galaxy (and near the orientation of the bar), it has recently encountered objects along its path that made it bend, so that the jet ends moved above and below the plane. In the north, such a barrier could be due to the merging nucleus that lies slightly north of the point where the jet leaves the bar. In the south, it is possible that the dust lanes trace the trail left behind by tidally stripped molecular clouds of the disrupted companion, and they cross the bar just outside the point where the jet bends toward southeast. CO emission is seen only in the bar, but in the south this is close to the point where the dust lanes cross the bar (Laine et al. 1999). Thus, these molecular clouds could have blocked the passage of the jet in the bar direction, deflecting it outside the bar. Simulations of jet deflection by a companion galaxy or by dense clouds have been made by Wang et al. (2000), who used a jet velocity around $4500 \mathrm{~km} \mathrm{~s}^{-1}$. These simulations did not attempt to model any specific observed system, but were of general nature. To get a deflection toward a certain direction, the impact must have taken place off-center with respect to the companion or molecular clouds. This is difficult to verify without knowing the exact jet speed and direction, as well as an accurate value for the pattern speed of the bar in which the companion and molecular clouds are trapped. The jet is rather variable in strength along its propagation direction and even has sections along its length that could be gaps. This could be due to an intermittent emission mechanism ("blobs"), or it could be related to the compression/deflection mechanism mentioned above.

It is unlikely that the structure that we see is an outflow or wind from the nucleus driven by the minor merger, since we do not see any evidence for the jetlike structure at any other than radio continuum wavelengths (a total lack of enhanced emission outside of the bar at other wavelengths). Furthermore, the feature is relatively narrow and has an almost perfectly aligned magnetic field, which would not be expected for an outflow. In fact, radio jets are predicted to exist in galaxies with relatively weak active galactic nuclei (AGNs), such as the one in NGC 7479, instead of torus outflows (Elitzur 2007).

Future VLBI observations have the potential of revealing the orientation of the jet near the nucleus and thus giving further clues to its origin (Scheuer \& Feiler 1996).

\subsection{Comparison to Other Nearby Spiral Galaxies with Large-Scale Jets}

The nearest (at around $7 \mathrm{Mpc}$ ) example of a spiral galaxy with a kiloparsec-scale radio jet is NGC 4258 (van der Kruit et al. 1972; Cecil et al. 1992, 2000). The radio jet extends to about 
$14 \mathrm{kpc}$ and has a spiral shape, but with the same pitch angle as the optical spiral arms (it winds in the same sense as the stellar spiral arms), unlike the anomalous radio continuum arms of NGC 7479. The jet in NGC 4258 has large RM values, even larger than in NGC 7479, possibly originating from a cocoon around the jet (Krause \& Löhr 2004). The intensities of the two jets at $3.5 \mathrm{~cm}$ are comparable, several millijanskys per beam at a resolution of about $12^{\prime \prime}$, and the highest intensities outside the nucleus are some distance away from the nucleus along the jet. The spectral indices are also comparable, about -0.7 to -0.8 in both galaxies. As the jet of NGC 4258 is narrower $(\leq 50 \mathrm{pc})$ than the partly resolved jet in NGC 7479, the total magnetic field as determined from the radio maps at $3^{\prime \prime}$ resolution is much stronger $(\simeq 300 \mu \mathrm{G})$. The degree of polarization in the jet of NGC 4258 is much higher (35\%-65\%) than in NGC 7479 , yielding stronger regular fields, consistent with the higher RMs in NGC 4258. Perhaps the most important difference is that the inner radio jet in NGC 4258 has its counterpart in $\mathrm{H} \alpha$ and X-ray emission (Courtés \& Cruvellier 1961; Cecil et al. 1995; Wilson et al. 2001), which is not seen for the bent jet in NGC 7479, and that there is a clear interaction with the dense ISM in the inner disk because $\mathrm{CO}$ line emission is seen along the inner jet (Krause et al. 1990, 2007; Krause \& Löhr 2004). The broader radio jet of NGC 7479 and its weaker magnetic field strength indicate weaker interaction with the ISM than in NGC 4258, which may explain the lack of any signature of the jet in NGC 7479 in other spectral regimes. The nucleus of NGC 4258 has been classified as LINER (Heckman 1980) or a relatively weak Seyfert 1.9 (e.g., Ho et al. 1997) and therefore has a similar nuclear activity level as NGC 7479.

The edge-on disk galaxy 0313-192 at a distance of close to $300 \mathrm{Mpc}$ has huge $(200 \mathrm{kpc})$ radio lobes of radio source type FR I that extend perpendicular to the disk (Ledlow et al. 2001). Only a small southern radio jet coming from the embedded AGN (Keel et al. 2006) has been detected, with a length of a few kiloparsecs. The jet is weak and its total flux density is only $729 \mu \mathrm{Jy}$ at $3.5 \mathrm{~cm}$ (Ledlow et al. 2001), corresponding to a radio power of $\approx 7 \times 10^{21} \mathrm{~W} \mathrm{~Hz}^{-1}$. There is a southern jetlike structure seen in the X-rays as well, of the same scale as the inner radio continuum jet (Keel et al. 2006). No such X-ray structure has been seen in NGC 7479. In comparison, the flux density of the southern jet of NGC 7479 is about $3 \mathrm{mJy}$ at $3.5 \mathrm{~cm}$ (radio power of $3.7 \times 10^{20} \mathrm{~W} \mathrm{~Hz}^{-1}$ ). The main differences compared to NGC 7479 are the existence of huge $100 \mathrm{kpc}$ scale radio lobes, the larger jet radio power, the faster jet, and less energy loss in the inner jet of $0313-192$. Due to its edge-on orientation, it is unknown whether 0313-192 has a bar or spiral arms.

NGC 3079 is another nearly edge-on $\left(i=84^{\circ}\right)$ spiral galaxy, but at a distance of only about $17 \mathrm{Mpc}$. It has large radio lobes perpendicular to the disk, extending to $3 \mathrm{kpc}$ above and below the galaxy plane (Duric \& Seaquist 1988). However, it possesses only a small parsec-scale nuclear radio jet, and the relation between the nuclear jet and the large-scale radio lobes is unclear. The radio lobes are most likely powered by a wind from the starburst nucleus, which, however, has also LINER and Seyfert 2 classifications and thus is a weakly active nucleus, similar to that in NGC 7479. There is no evidence for a strong starburst nucleus or a wind driven by a starburst in NGC 7479. The main difference between NGC 3079 and NGC 7479 is the lack of any kiloparsec-scale radio jet in NGC 3079. Therefore, the radio feature in NGC 7479 is most likely driven by the active nucleus, whereas the radio structure in NGC 3079 is driven by the central starburst. Other radio lobes/outflow features among nearby edge-on spiral galaxies are seen, e.g., in the Sombrero galaxy NGC 4594 and in NGC 4235 (Gallimore et al. 2006).

The nearby Circinus galaxy (distance $d \approx 4 \mathrm{Mpc}$ ) has bipolar radio lobes (Harnett et al. 1990), a 10 pc scale nuclear jetlike feature emanating from the Seyfert 2 nucleus, aligned with the radio lobes (Elmouttie et al. 1998), and a parsec-scale nuclear outflow (Greenhill et al. 2003). Again, no kiloparsec-scale radio jet is seen.

In summary, starburst galaxies, such as M82 (e.g., Seaquist \& Odegard 1991) and NGC 3079 (above), possess diffuse radio lobes that are usually oriented perpendicular to the galaxy disk. Colbert et al. (1996) found that several nearby edge-on Seyfert galaxies also have kiloparsec-scale diffuse radio continuum morphologies that are not always aligned along the galaxy minor axis. NGC 7479 does not possess a strong nuclear starburst, has a $15 \mathrm{kpc}$ scale, relatively well collimated radio jet, and therefore differs from both the starburst galaxies and Seyferts with diffuse kiloparsec-scale radio continuum structures. Thus, it represents a previously unknown type of galaxy with a possibly interactiontriggered, $15 \mathrm{kpc}$ scale, most likely short-lived radio jet that is radio bright due to its location very close to the disk plane.

\section{SUMMARY}

We have examined the nature of the anomalous $15 \mathrm{kpc}$ scale jetlike feature in the nearby barred spiral galaxy NGC 7479 with radio continuum observations at $3.5 / 3.6,6,18$, and $22 \mathrm{~cm}$. We have found strong evidence to support the jetlike nature of this feature and its origin in the nucleus of the galaxy. Most importantly, we have shown that the large-scale radio properties of NGC 7479 differ from any other previously mapped disk galaxy in a fundamental way. Therefore, NGC 7479 provides us a unique opportunity to study interaction-triggered, short-lived, $15 \mathrm{kpc}$ scale radio jets and large-scale magnetic fields in disk galaxies.

Our observations suggest that the jet of NGC 7479 is located very close to the disk plane, and thus it causes compression of the interstellar magnetic fields and acceleration of cosmic-ray particles, resulting in an exceptionally radio-bright jet. However, the magnetic field in the jet of NGC 7479 is about 10 times weaker than in NGC 4258, which could account for the lack of associated extended $\mathrm{H} \alpha$ and the weak X-ray emission. The polarized radio emission from the jet serves as a background against which we can measure, with the help of Faraday rotation and depolarization, the structure of the regular magnetic field in the foreground disk of NGC 7479 with unprecedented precision. This technique has revealed multiple magnetic field reversals in the disk. We have produced the most detailed study of spiral disk galaxy magnetic fields using the jet in NGC 7479.

A more detailed summary of our main results is given below:

1. The total magnetic field strength is $\simeq 35 \mu \mathrm{G}$ in the inner jet and $\simeq 40 \mu \mathrm{G}$ in the outer jet, which limits the lifetime of cosmicray electrons to about $10^{6} \mathrm{yr}$.

2. We find that the magnetic field is closely aligned along the radio continuum jet feature throughout its extent.

3. The degree of polarization at $3.5 \mathrm{~cm}$ is $6 \%-8 \%$ along the jet, and remarkably constant, which is consistent with helical field models for jets.

4. We can exclude the possibility that the observed Faraday rotation (RM) between 3.5 and $6 \mathrm{~cm}$ and the depolarization (DP) between 6 and $22 \mathrm{~cm}$ occur in the jet itself because we do not see an anticorrelation between RM and DP (as would be expected if synchrotron emission and Faraday rotation occur in the same 
volume) and no $\mathrm{H} \alpha$ and only weak X-ray emission is detected along the jet.

5. The observed Faraday rotation and depolarization are consistent with magnetoionic gas in the disk of the galaxy, between us and the jet, with a regular field strength of about $8 \mu \mathrm{G}$ and thermal electron densities of $\simeq 0.06 \mathrm{~cm}^{-3}$ near the bar and $\simeq 0.03 \mathrm{~cm}^{-3}$ outside the bar. The jet cannot be bent out of the disk scale by more than a few degrees, and its true total length is about $15 \mathrm{kpc}$. Some fraction of the Faraday effects may be generated in a cocoon of ionized gas and regular magnetic fields around the jet.

6. The regular magnetic field in the disk of the galaxy has multiple reversals on scales of 1-2 kpc, indicating field loops stretched by a shearing gas flow in the bar potential of the galaxy or by gas flows related to the merger event.

7. Faraday dispersion by cells of random field in the foreground disk can explain the depolarization data. This model can also account for the total depolarization of the northern jet at $22 \mathrm{~cm}$ if this part of the jet is located behind the galaxy disk.

8. The "leading" spiral morphology of the jet, opposite to the trailing sense of the stellar and gaseous spiral arms, may be due to precession, driven by a precessing accretion disk near the nucleus or a binary black hole system. Either one of these could be a consequence of a recent minor merger event in this system. Alternatively, jet bending could be due to an off-center collision with pieces of the companion (its nucleus and dense molecular clouds) that happened to be in the path of the jet.

9. As the jet interacts with the ISM in the disk, the jet must propagate at a speed of more than $5000 \mathrm{~km} \mathrm{~s}^{-1}$ to avoid bending by differential rotation; hence, it is most likely less than about $10^{6} \mathrm{yr}$ old, and thus a very recent feature. This may explain why such jets are not commonly seen in nearby disk galaxies.

10. The jet of NGC 7479 is the second case of a large jet located almost in the plane of the host galaxy. Compared to
NGC 4258, the jet in NGC 7479 is broader, less polarized, hosts a weaker magnetic field, and is bent in a sense opposite to that of the spiral arms. Both jets are radio bright, probably due to interaction with the dense ISM gas of the disk, lose most of their energy within the disk, and hence cannot form outer radio lobes.

11. More sensitive $\mathrm{H} \alpha$ and $\mathrm{X}$-ray observations are needed to search for signs of interaction between the jet and the ISM.

We are grateful to Vladimir Shoutenkov for help with the data reduction and to Matthias Ehle for providing the X-ray image and for model fitting of the X-ray data. We thank Marita Krause for a critical reading of the manuscript and many useful comments on an earlier version of this paper. We thank Max Camenzind, Christian Kaiser, and Robert Laing for very useful discussions on jet triggering mechanisms and Bruce Elmegreen, Arieh Königl, and Tim Heckman for enlightening discussions on the jet feature. We thank the anonymous referee for many useful comments. The National Radio Astronomy Observatory is a facility of the National Science Foundation operated under cooperative agreement by Associated Universities, Inc. The Effelsberg $100 \mathrm{~m}$ telescope is operated by the Max-Planck-Institut für Radioastronomie in Bonn on behalf of the Max-Planck-Gesellschaft (MPG). This research has made use of the NASA/IPAC Extragalactic Database (NED), which is operated by the Jet Propulsion Laboratory, California Institute of Technology, under contract with the National Aeronautics and Space Administration. This work is based in part on observations made with the Spitzer Space Telescope, which is operated by the Jet Propulsion Laboratory, California Institute of Technology under a contract with NASA.

Facilities: VLA, Effelsberg, Spitzer(IRAC), XMM(EPIC)
Beck, R. 2005, in Cosmic Magnetic Fields, ed. R. Wielebinski \& R. Beck (Berlin: Springer), 41

. 2007, A\&A, 470, 539

Beck, R., Fletcher, A., Shukurov, A., Snodin, A., Sokoloff, D. D., Ehle, M., Moss, D., \& Shoutenkov, V. 2005, A\&A, 444, 739

Beck, R., \& Krause, M. 2005, Astron. Nachr., 326, 414

Beck, R., Poezd, A. D., Shukurov, A., \& Sokoloff, D. D. 1994, A\&A, 289, 94

Beck, R., Shoutenkov, V., Ehle, M., Harnett, J. I., Haynes, R. F., Shukurov, A., Sokoloff, D. D., \& Thierbach, M. 2002, A\&A, 391, 83

Begelman, M. C., Blandford, R. D., \& Rees, M. J. 1980, Nature, 287, 307

Bicknell, G. V., Cameron, R. A., \& Gingold, R. A. 1990, ApJ, 357, 373

Blackman, C. P. 1983, MNRAS, 202, 379

Bridle, A. H., \& Perley, R. H. 1984, ARA\&A, 22, 319

Burbidge, E. M., Burbidge, G. R., \& Prendergast, K. H. 1960, ApJ, 132, 654

Cecil, G., Morse, J. A., \& Veilleux, C. 1995, ApJ, 452, 613

Cecil, G., Wilson, A. S., \& Tully, R. B. 1992, ApJ, 390, 365

Cecil, G., et al. 2000, ApJ, 536, 675

Colbert, E. J. M., Baum, S. A., Gallimore, J. F., O’Dea, C. P., \& Christensen, J. A. 1996, ApJ, 467, 551

Courtés, G., \& Cruvellier, P. 1961, CR Acad. Sci. Paris, 253, 218

Cox, P., \& Downes, D. 1996, ApJ, 473, 219

Daigle, A., \& Roy, J. R. 2001, ApJ, 552, 144

Devereux, N. A. 1989, ApJ, 346, 126

Duric, N., \& Seaquist, E. R. 1988, ApJ, 326, 574

Elitzur, M. 2007, in ASP Conf. Ser. 373, The Central Engine of Active Galactic Nuclei, ed. L. C. Ho \& J.-M. Wang (San Francisco: ASP), 415

Elmouttie, M., Haynes, R. F., Jones, K. L., Sadler, E. M., \& Ehle, M. 1998, MNRAS, 297, 1202

Gallimore, J. F., Axon, D. J., O’Dea, C. P., Baum, S. A., \& Pedlar, A. 2006, AJ, 132,546

Garrington, S. T., Leahy, J. P., Conway, R. G., \& Laing, R. A. 1988, Nature, 331,147

Greenhill, L. J., Henkel, C., Becker, R., Wilson, T. L., \& Wouterloot, J. G. A. 1995a, A\&A, 304, 21
Greenhill, L. J., Jiang, D. R., Moran, J. M., Reid, M. J., Lo, K. Y., \& Claussen, M. J. 1995b, ApJ, 440, 619

Greenhill, L. J., et al. 2003, ApJ, 590, 162

Harnett, J. I., Whiteoak, J. B., Reynolds, J. E., Gardner, F. F., \& Tzioumis, A. 1990, MNRAS, 244, 130

Heckman, T. M. 1980, A\&A, 87, 152

Herrnstein, J. R., Moran, J. M., Greenhill, L. J., Diamond, P. J., Miyoshi, M., Nakai, N., \& Inoue, M. 1997, ApJ, 475, L17

Herrnstein, J. R., et al. 1999, Nature, 400, 539

Ho, L. C., Filippenko, A. V., \& Sargent, W. L. W. 1997, ApJS, 112, 315

Ho, L. C., \& Ulvestad, J. S. 2001, ApJS, 133, 77

Hummel, E., Krause, M., \& Lesch, H. 1989, A\&A, 211, 266

Irwin, J. A., \& Seaquist, E. R. 1988, ApJ, 335, 658

Johnston-Hollitt, M., Hollitt, C. P., \& Ekers, R. D. 2004, in The Magnetized Interstellar Medium, ed. B. Uyaníker, W. Reich, \& R. Wielebinski (Katlenburg: Copernicus), 13

Keel, W. C. 1983, ApJS, 52, 229

Keel, W. C., White, R. E., Owen, F. N., \& Ledlow, M. J. 2006, AJ, 132, 2233

Krause, F., \& Beck, R. 1998, A\&A, 335, 789

Krause, M., Cox, P., Garcia-Barreto, J. A., \& Downes, D. 1990, A\&A, 233, L1

Krause, M., Fendt, C., \& Neininger, N. 2007, A\&A, 467, 1037

Krause, M., \& Löhr, A. 2004, A\&A, 420, 115

Laine, S. 1996, Ph.D. thesis, Univ. Florida

Laine, S., \& Gottesman, S. T. 1998, MNRAS, 297, 1041

Laine, S., \& Heller, C. H. 1999, MNRAS, 308, 557

Laine, S., Kenney, J. D. P., Yun, M. S., \& Gottesman, S. T. 1999, ApJ, 511, 709

Laine, S., Kotilainen, J. K., Reunanen, J., Ryder, S. D., \& Beck, R. 2006, AJ, 131,701

Laing, R. A. 1981, ApJ, 248, 87

Ledlow, M. J., Owen, F. N., Yun, M. S., \& Hill, J. M. 2001, ApJ, 552, 120

Lu, J. F. 1990, A\&A, 229, 424

Maloney, P. R., Begelman, M. C., \& Pringle, J. E. 1996, ApJ, 472, 582

Martin, P., Leliévre, M., \& Roy, J.-P. 2000, ApJ, 538, 141

Merritt, D., \& Ekers, R. D. 2002, Science, 297, 1310 
Miyoshi, M., Moran, J., Herrnstein, J., Greenhill, L., Nakai, N., Diamond, P., \& Inoue, M. 1995, Nature, 373, 127

Moss, D., Snodin, A. P., Englmaier, P., Shukurov, A., Beck, R., \& Sokoloff, D. D. 2007, A\&A, 465, 157

Nagar, N. M., Falcke, H., \& Wilson, A. S. 2005, A\&A, 435, 521

Panessa, F., Bassani, L., Cappi, M., Dadina, M., Barcons, X., Carrera, F. J., Ho, L. C., \& Iwasawa, K. 2006, A\&A, 455, 173

Quillen, A., Frogel, J. A., Kenney, J. D. P., Pogge, R. W., \& DePoy, D. L. 1995, ApJ, 441, 549

Rees, M. J., Begelman, M. C., Blandford, R. D., \& Phinney, E. S. 1982, Nature, 295,17

Sandage, A., \& Tammann, G. A. 1987, A Revised Shapley-Ames Catalog of Bright Galaxies (2nd ed.; Washington DC: Carnegie Inst.)

Sanders, R. H. 1982, in Extragalactic Radio Sources, ed. D. S. Heeschen \& C. M. Wade (Dordrecht: Kluwer), 145
Saraiva, M. F., \& Benedict, G. F. 2003, A\&A, 409, 899 Scheuer, P. A. G., \& Feiler, R. 1996, MNRAS, 282, 291

Seaquist, E. R., \& Odegard, N. 1991, ApJ, 369, 320

Sokoloff, D. D., Bykov, A. A., Shukurov, A., Berkhuijsen, E. M., Beck, R., \& Poezd, A. D. 1998, MNRAS, 299, 189 (erratum 303, 207)

van der Kruit, P. C., Oort, J. H., \& Mathewson, D. S. 1972, A\&A, 21, 169

Vogler, A., \& Pietsch, W. 1999, A\&A, 352, 64

Walker, I. R., Mihos, J. C., \& Hernquist, L. 1996, ApJ, 460, 121

Wang, Z., Wiita, P. J., \& Hooda, J. S. 2000, ApJ, 534, 201

Wielebinski, R. 2005, in Cosmic Magnetic Fields, ed. R. Wielebinski \& R. Beck (Berlin: Springer), 89

Wilson, A. S., Yang, Y., \& Cecil, G. 2001, ApJ, 560, 689

Young, J. S., Xie, S., Kenney, J. D. P., \& Rice, W. L. 1989, ApJS, 70, 699 